- RAM, REV. ADM. MACKENZIE, 16(3), Edição Especial • SÃO PAULO, SP • MAIO/JUN. 2015 • ISSN 1518-6776 (impresso) • ISSN 1678-6971 (on-line) • http://dx.doi.org/10.1590/1678-69712015/administracao.v16n3p136-170. Submissão: 5 nov. 2014. Aceitação: 3 mar. 2015.

Sistema de avaliação: às cegas dupla (double blind review). UNIVERSIDADE PRESBITERIANA MACKENZIE. Silvio Popadiuk (Ed.),

Thais Elaine Vick, Denise Del Prá Netto Machado, Fernando Gomes de Paiva Junior e Cleber Carvalho de Castro (Ed. convidados), p. 136-170.

\title{
1 ANÁLISE DOS ARTIGOS QUALITATIVOS EMPÍRICOS SOBRE MÉTODOS, TÉCNICAS E FERRAMENTAS PARA INOVAÇÃO ${ }^{\mathrm{I}}$
}

GUSTAVO TOMAZ BUCHELE

Graduado em Administração pelo Centro de Ciências da Administração e Socioeconômicas da Universidade do Estado de Santa Catarina (Esag-Udesc). Mestrando em Engenharia e Gestão do Conhecimento do Centro Tecnológico da Universidade Federal de Santa Catarina (UFSC).

Campus Universitário Reitor João David Ferreira Lima, Trindade, Florianópolis - SC - Brasil - CEP 88040-900 E-mail: gustavotb.adm@gmail.com

PIERRY TEZA

Mestre em Engenharia de Produção e Sistemas pelo Centro Tecnológico da Universidade Federal de Santa Catarina (UFSC).

Doutorando em Engenharia e Gestão do Conhecimento do Centro Tecnológico da Universidade Federal de Santa Catarina (UFSC).

Campus Universitário Reitor João David Ferreira Lima, Trindade, Florianópolis - SC - Brasil - CEP 88040-900

E-mail: pierryteza@gmail.com

GERTRUDES APARECIDA DANDOLINI

Doutora em Engenharia de Produção e Sistemas pelo Centro Tecnológico da Universidade Federal de Santa Catarina (UFSC).

Professora do Departamento de Engenharia e Gestão do Conhecimento da Universidade Federal de Santa Catarina (UFSC).

Campus Universitário Reitor João David Ferreira Lima, Trindade, Florianópolis - SC - Brasil - CEP 88040-900

E-mail:ggtude@gmail.com

JOÃO ARTUR DE SOUZA

Doutor em Engenharia de Produção e Sistemas pelo Centro Tecnológico da Universidade Federal de Santa Catarina (UFSC).

Professor do Departamento de Engenharia e Gestão do Conhecimento da Universidade Federal de Santa Catarina (UFSC).

Campus Universitário Reitor João David Ferreira Lima, Trindade, Florianópolis - SC - Brasil - CEP 88040-900

E-mail: jartur@gmail.com

I Os autores agradecem aos avaliadores da Revista de Administração Mackenzie a contribuição significativa para a melhoria do presente trabalho.

Este artigo pode ser copiado, distribuído, exibido, transmitido ou adaptado desde que citados, de forma clara e explícita, o nome da revista, a edição, o ano, e as páginas nas quais o artigo foi publicado originalmente, mas sem sugerir que a RAM endosse a reutilização do artigo. Esse termo de licenciamento deve ser explicitado para os casos de reutilização ou distribuição para terceiros. Não é permitido o uso para fins comerciais. 


\section{RESUMO}

A inovação tem sido considerada um elemento essencial para criar vantagem competitiva de longo prazo nas empresas. Porém, estimular e apoiar o processo de inovação ainda é um desafio. Por sua vez, o efetivo uso de métodos, técnicas e ferramentas para inovação pode ser um fator importante para apoiar o processo da gestão da inovação, aumentando suas chances de sucesso. Nesse sentido, o estudo aqui apresentado consistiu em uma análise dos artigos qualitativos empíricos relacionados à difusão e adoção de métodos, ferramentas e técnicas para inovação. Os artigos analisados foram obtidos por meio de um levantamento sistemático realizado a partir de duas bases de dados: Scopus e Web of Science. Esse levantamento sistemático consistiu em sete passos: identificação das palavras-chave, buscas nas bases de dados, filtragem das publicações, padronização, classificação dos artigos, inclusão de publicações relevantes e análise dos artigos. Como resultado, obteve-se um corpus de dez publicações (nove disponíveis na íntegra e uma somente resumo), nas quais, inicialmente, se identificaram os principais artigos, autores, países e periódicos que mais publicaram e as palavras-chave mais utilizadas. Posteriormente, a partir da análise realizada, possibilitou-se um panorama das pesquisas qualitativas empíricas relacionadas ao tema, dada a importância dos MTF-Is para o processo de inovação das empresas. Destaca-se que ainda são escassos os trabalhos nessa temática com a abordagem utilizada nesta pesquisa, além de os resultados encontrados não serem convergentes. Verificou-se a grande diversidade de métodos, técnicas e ferramentas para inovação utilizados pelas empresas estudadas. Contudo, foi possível identificar que a utilização de MTF-Is é importante para aumentar a competitividade e principalmente resolver problemas nas organizações. Observou-se a necessidade de mais estudos com o objetivo de sistematizar a tomada de decisão acerca da escolha de um MTF-I para um determinado contexto organizacional. Dessa forma, espera-se que este trabalho possa servir de base para pesquisas futuras acerca do tema. 


\section{PALAVRAS-CHAVE}

Inovação. Métodos, técnicas e ferramentas para inovação. MTF-Is. Levantamento sistemático. Métodos qualitativos.

\section{INTRODUÇÃO}

A crescente importância do conhecimento como fator de produção e como determinante para a inovação pode ser explicada pela acumulação contínua de conhecimento técnico ao longo do tempo e pelo uso de tecnologias de comunicação que permitem que o conhecimento seja diponibilizado rapidamente em uma grande escala (Hidalgo \& Albors, 2008). Tendo em vista que os mercados se tornaram altamente competitivos e os clientes muito exigentes (Blocker, Flint, Myers, $\&$ Slater, 20II), há a necessidade de as empresas inovarem em diversos aspectos para atender às necessidades desses clientes (Mattar, Oliveira, Queiroz, \& Motta, 2009). Nesse sentido, as organizações estão sob crescente pressão competitiva para manter a fatia de mercado, aumentar a gama de produtos, melhorar a eficiência e reduzir custos, sendo a inovação o processo que pode levá-las a alcançar esses objetivos (Flynn, Dooley, O'Sullivan, \& Cormican, 2003). Assim, as constantes demandas e mudanças do ambiente exigem uma constante adaptação das organizações por meio da inovação, que pode ser realizada em relação a produtos, serviços, operações, processos e pessoas (Baregheh, Rowley, \& Sambrook, 2009).

A inovação é vital para a vantagem competitiva de longo prazo das empresas. No entanto, motivar e estimular a inovação continua a ser um desafio para a maioria delas (Tian \& Wang, 20I4). Baregheh et al. (2009) afirmam que a inovação é o processo de várias etapas por meio do qual as organizações transformam ideias em produtos novos/melhorados, serviços ou processos, a fim de avançar, competir e diferenciar-se com sucesso em seu mercado. Ela ainda pode ser definida como o processo pelo qual novas ideias, objetos ou práticas são criados, desenvolvidos, implementados e difundidos (Walker, 2006).

Dada a importância para as organizações, diversas pesquisas têm focado no processo de inovação, principalmente buscando estudar formas de melhorá-lo como um todo. Essas pesquisas iniciaram com um enfoque maior na área de desenvolvimento de produtos (com ênfase em bens físicos), e, ao longo do tempo, os esforços têm sido transferidos para a área de inovação, de forma a abranger outros resultados do processo, como novos e/ou melhorados serviços e processos.

Uma dimensão de decisões importantes a serem tomadas em relação ao processo de inovação em geral refere-se a quais abordagens utilizar ao longo 
do processo. Essas abordagens suportam o entendimento, a análise, decisão e ação ao longo do processo de inovação (Phaal, Kerr, Oughton, \& Probert, 20I2). Essas abordagens, aqui chamadas de métodos, técnicas e ferramentas para inovação (MTF-I), incluem brainstorming, análise morfológica, grupo focal, teste de conceito, cenários e retorno sobre o investimento (Nijssen \& Lieshout, I995; D'Alvano \& Hidalgo, 20I2). Diversos estudos têm verificado que a adoção efetiva de MTF-Is é um fator importante para o desempenho do processo de inovação (Nijssen \& Frambach, 2000; Chai \& Xin, 2006; Graner \& Mißler-Behr, 20I2).

Embora o estudo dos MTF-Is seja importante, principalmente para fomentar seu uso e consequentemente melhorar a efetividade do processo de inovação, o tema carece de mais pesquisas. Ainda, verificam-se o baixo uso da abordagem qualitativa em relação à abordagem quantitativa de pesquisa e, a partir disso, a dificuldade de entender com profundidade diversos aspectos relativos ao uso de MTF-I, como barreiras e facilitadores da difusão e adoção destes.

Nesse sentido, a partir da necessidade de entender de que forma as pesquisas qualitativas empíricas vêm abordando a temática MTF-I, apresentam-se neste trabalho as contribuições acadêmicas ao tema, ou seja, avaliação crítica e imparcial dos MTF-Is existentes, identificação de lacunas no campo, geração de novos dados para aprofundamento da compreensão do tema e possibilidade de utilização desses novos dados para auxiliar o desenvolvimento de novos MTF-I (Brady et al., I997). Assim, estabeleceu-se a seguinte pergunta de pesquisa:

- Como a temática MTF-I vem sendo estudada empiricamente com a utilização da abordagem qualitativa?

Para responder a essa questão, utilizou-se um levantamento sistemático em duas bases de dados científicas, seguido da categorização dos trabalhos levantados e da análise daqueles cujas pesquisas utilizaram a abordagem qualitativa empírica com foco em difusão e adoção de MTF-I, uma vez que se pretendeu estudar a temática sobre um ponto de vista mais específico, excluindo, por exemplo, artigos que tratam da proposição apenas de um novo MTF-I. Adoção refere-se à decisão da empresa em usar um MTF-I no seu processo de inovação ou rejeitá-lo, e difusão está relacionada ao número cumulativo de empresas que têm adotado um determinado MTF-I ao longo do tempo (Chai \& Xin, 2006).

Assim, este trabalho está estruturado em cinco seções: a primeira e presente seção corresponde à introdução do artigo. Na segunda seção, são levantados conceitos importantes relacionados ao tema. Na terceira, detalham-se os procedimentos metodológicos utilizados no levantamento dos artigos. A quarta seção apresenta a análise dos artigos qualitativos empíricos com foco em difusão e adoção de MTF-I. Finalmente, na quinta e última seção, apresentam-se as considerações finais e 
explicitam-se os pontos que poderão ser abordados em estudos futuros. Ao final, são apresentadas as referências utilizadas como aporte teórico para este trabalho.

\section{REFERENCIAL TEÓRICO}

Muitos MTF-Is têm sido desenvolvidos nas últimas décadas com o objetivo de melhorar o processo de desenvolvimento de novos produtos e torná-lo mais gerenciável (Mahajan \& Wind, I992; Nijssen \& Lieshout, I995; Nijssen \& Frambach, 2000; Thia, Chai, Bauly, \& Xin, 2005; Chai \& Xin, 2006; Yeh et al., 2010). Além disso, a análise dos trabalhos relacionados evidencia uma divergência de termos, os quais não são diferenciados pelos autores no que concerne aos aspectos conceituais e operacionais (Phaal et al., 2012). Nesse sentido, diversas terminologias são utilizadas para fazer referência à abordagem MTF-I, como: ferramentas (Coulon, Ernst, Lichtenthaler, \& Vollmoeller, 2009; Nijssen \& Frambach, 2000; Hidalgo \& Albors, 2008); ferramentas e técnicas (Fleisher, 2006; Igartua, Garrigós, \& Hervas-Oliver, 20I0); métodos (Lichtenthaler, 2005); modelos e métodos (Nijssen \& Lieshout, I995). Poucos trabalhos abordam a questão da terminologia (por exemplo, Shehabuddeen, Probert, Phaal, \& Platts, I999). Aqui serão utilizados os termos métodos, técnicas e ferramentas sem inicialmente fazer distinção entre eles, considerando que podem ser um documento, framework, procedimento, sistema ou método, de modo a permitir que a organização alcance ou clarifique um objetivo (Brady et al., I997).

Os MTF-Is são considerados os meios fundamentais para aumentar a competitividade e podem ser definidos como o conjunto de métodos, técnicas e ferramentas que suportam o processo de inovação nas empresas, com o propósito de ajudá-las de forma sistemática a atender aos novos desafios do mercado (Phaal, Farrukh, \& Probert, 2006; Hidalgo \& Albors, 2008; Igartua et al., 20I0; D'Alvano \& Hidalgo, 20I2). O uso efetivo de MTF-Is tem sido um importante elemento na gestão do processo de inovação (Thia et al., 2005), uma que vez eles facilitam a habilidade de uma organização em introduzir apropriadamente novas tecnologias em produtos e processos e as mudanças necessárias à organização (Hidalgo \& Albors, 2008). Eles podem ajudar as organizações a gerir a inovação, adaptar-se às novas circunstâncias e enfrentar os desafios de mercado de uma forma sistemática (Igartua et al., 2010).

Chiesa e Masella (1996) afirmam, em seu modelo de auditoria do processo de inovação tecnológica, que o efetivo uso de MTF-I é um dos três facilitadores desse processo, em conjunto com o desenvolvimento de recursos humanos e físicos e liderança e direção da alta gestão. Embora eles não possam garantir o sucesso, o uso de MTF-I pode servir para identificar problemas sistematicamente, complementando os esforços da organização (Cooper \& Kleinschmidt, I986). 
Assim, a sua utilização é destinada a fases específicas do processo de inovação (Mahajan \& Wind, I992; Nijssen \& Lieshout, I995; Nijssen \& Frambach, 2000; Chai \& Xin, 2006). Entretanto, alguns MTF-I podem ser úteis para atingir vários objetivos e ser utilizados em diversos estágios desse processo, como brainstorming e desdobramento da função qualidade (quality function deployment - QFD) (Nijssen \& Lieshout, I995; Nijssen \& Frambach, 2000; Chai \& Xin, 2006).

\section{PROCEDIMENTOS METOdOLÓGICOS}

A exemplo de Graner e Mißler -Behr (20I2), esta revisão enfoca todos aqueles trabalhos publicados em periódicos que têm MTF-Is utilizados em estudos empíricos até a data. Dessa forma, o estudo aqui apresentado foi composto por duas etapas distintas: I. levantamento dos artigos relacionados aos MTF-Is; 2. análise dos artigos qualitativos empíricos relacionados a essa abordagem. Na primeira etapa, além do levantamento dos artigos, buscou-se uma análise geral deles de forma a proporcionar um panorama das pesquisas nessa área, bem como a identificação dos artigos qualitativos empíricos, foco do estudo aqui apresentado. Essa etapa foi executada por meio das seguintes atividades: identificação das palavras-chave, buscas nas bases de dados, filtragem das publicações, padronização, classificação dos artigos, inclusão de publicações relevantes e análise dos artigos.

- Identificação das palavras-chave: inicialmente, para a identificação das palavras-chave a serem utilizadas na busca, optou-se por verificar as palavras-chave e os termos mais utilizados pelas publicações relacionadas aos MTF-Is. Assim, realizou-se, na base Scopus, uma busca inicial com os seguintes termos combinados com a palavra innovation: method, technique e tool. A busca foi realizada em títulos, resumos e palavras-chave. Utilizaram-se os termos em inglês para proporcionar maior abrangência à busca. Encontraram-se dezenas de milhares de artigos, o que poderia inviabilizar a análise. Ainda, verificou-se que, em alguns casos, os MTF-Is são tratados em campos como desenvolvimento de novos produtos e inteligência tecnológica, e essas expressões foram citadas nos títulos dos artigos, sem a menção do termo innovation. Assim, com o propósito de viabilizar a análise, optou-se por realizar as buscas apenas nos títulos dos artigos. Porém, para que artigos relevantes não fossem perdidos, optou-se por ampliar as palavras-chave da busca. Nesse sentido, definiram-se, para a busca nas bases de dados, as seguintes expressões combinadas isoladamente com os termos method, technique e tool: front end, innovation, product development, technology development, technology intelligence e technology management.

- Buscas nas bases de dados: com relação à escolha das bases, selecionaram-se duas bases eletrônicas: Scopus e Web of Science. A escolha dessas bases deu-se por 
elas serem, na área de gestão de negócios, reconhecidas pela qualidade das publicações armazenadas. Além disso, optou-se por realizar a busca em duas bases, na tentativa de obter inicialmente uma maior abrangência para o levantamento. As buscas foram realizadas no mês de janeiro de 20I4. Optou-se pela utilização apenas de publicações em periódicos, uma vez que já estão avaliados pelos pares e assim constituem fonte mais confiável para análise. Em função da parametrização disponível em cada base, as buscas foram realizadas de forma diferente, porém com ajuda do software EndNote ${ }^{\circledR}$, no qual as referências foram importadas e os resultados filtrados, de forma a obter o mesmo critério para duas bases. Nesse sentido, obtiveram-se I.582 publicações.

- Filtragem das publicações: no EndNote ${ }^{\circledR}$, as publicações foram reunidas, e, em seguida, eliminaram-se aquelas duplicadas entre bases, incluindo as que não eram artigos de periódicos. Após, identificaram-se os artigos que não eram relevantes para a pesquisa. Esse processo foi realizado com base na leitura dos títulos e resumos de cada publicação e eventualmente por meio do artigo completo - essa última opção foi utilizada nos casos em que o resumo não estava disponível ou suficientemente claro. Desse processo resultaram 203 artigos.

- Padronização: como os dados dos artigos vieram de duas bases de dados que possuem padrões distintos, para tornar viável a análise bibliométrica, foi realizada a padronização das informações. Essa padronização procurou não apenas igualar nomes dos autores e periódicos, mas também eliminar algumas inconsistências encontradas em função de erros de cadastro nas bases, como um nome incorreto de um periódico ou autor.

- Classificação dos artigos: por meio de uma análise aprofundada dos artigos, realizou-se a classificação deles segundo quatro critérios: I. a quantidade de MTF-I (um; mais de um); 2. a fonte de dados predominante (empírico; teórico); 3. a abordagem de pesquisa predominante (qualitativa; quantitativa); 4. a temática (difusão e adoção de MTF-I; outras). Essa última classificação foi necessária, uma vez que o foco desta pesquisa são os estudos empíricos sobre difusão e adoção de MTF-Is, conforme já mencionado. A classificação em relação à quantidade de MTF-I, essa se justifica porque a análise dos artigos evidenciou que aqueles que tratavam de mais de dois MTF-Is tinham uma abordagem predominantemente genérica sobre o estudo de MTF-I, em geral com foco na difusão e/ou adoção destes, diferentemente daquelas do segundo grupo, cujo foco é, em geral, a proposição e/ou aplicação de um MTF-I específico. Do resultado dessa análise, os artigos foram classificados em quatro grupos: A (dois ou mais MTF-Is; empíricos; com foco na temática difusão e/ou adoção; utilizando abordagem qualitativa), B (dois ou mais MTF-Is; empíricos; com foco na temática difusão e/ou adoção; utilizando abordagem quantitativa), C (dois ou mais MTF-Is; empíricos; não trata de 
adoção ou difusão; utilizando as abordagens qualitativa e/ou quantitativa), D (dois ou mais MTF-Is; teóricos; com foco na temática difusão e/ou adoção; todas as abordagens), E (dois ou mais MTF-Is; não trata de adoção ou difusão; todas as abordagens) e F (um MTF-I; teóricos e empíricos; todas as temáticas; todas as abordagens). O Quadro I apresenta os grupos e critérios utilizados na classificação dos artigos.

- Inclusão de publicações relevantes aos grupos $A, B$ e $D$ : de forma a evitar que artigos importantes para os grupos foco da pesquisa (grupos A, B e D) não fossem analisados em função de não estarem presentes em nenhuma das bases utilizadas, analisaram-se as referências das publicações disponíveis em texto completo procurando por artigos relevantes. Também se analisaram as referências dos artigos do grupo D, já que tratam também de difusão e/ou adoção de MTF-I. Especificamente, procuraram-se apenas artigos de periódico, conforme critério mencionado anteriormente. Dessa análise não foram identificados artigos qualitativos empíricos (grupo A) ou teóricos (grupo D) que já não estivessem sido levantados, porém identificaram-se mais quatro artigos quantitativos empíricos (grupo B). Dessa forma, obteve-se uma base de 207 artigos. Assim, as frequências obtidas por grupo podem ser observadas no Quadro I.

\section{QUADRO I}

\section{SEGMENTAÇÃO DOS ARTIGOS PROVENIENTES}

DAS BASES DE DADOS

\begin{tabular}{|c|c|c|c|c|c|}
\hline $\begin{array}{l}\text { QUANT. } \\
\text { DE MTF-IS }\end{array}$ & FONTE DE DADOS & TEMÁTICA & ABORDAGEM & GRUPO & QUANT. \\
\hline \multirow{5}{*}{$\begin{array}{c}\text { Dois ou } \\
\text { mais }\end{array}$} & \multirow{3}{*}{$\begin{array}{l}\text { Empíricos ou } \\
\text { predominantemente } \\
\text { empíricos }\end{array}$} & \multirow{2}{*}{$\begin{array}{c}\text { Predominantemente } \\
\text { sobre difusão e/ou } \\
\text { adoção de MTF-I }\end{array}$} & $\begin{array}{l}\text { Qualitativa ou } \\
\text { predominantemente } \\
\text { qualitativa (mista) }\end{array}$ & $\begin{array}{c}\text { A } \\
\text { (qualitativos } \\
\text { empíricos) }\end{array}$ & $\begin{array}{c}10 \text { (base) } \\
0 \text { (ref) }\end{array}$ \\
\hline & & & $\begin{array}{l}\text { Quantitativa ou } \\
\text { predominantemente } \\
\text { quantitativa (mista) }\end{array}$ & $\begin{array}{c}\text { B } \\
\text { (quantitativos } \\
\text { empíricos) }\end{array}$ & $\begin{array}{c}14 \text { (base) } \\
4 \text { (ref) }\end{array}$ \\
\hline & & Outras temáticas & Todas as abordagens & C & 7 \\
\hline & \multirow{2}{*}{$\begin{array}{c}\text { Teóricos ou } \\
\text { predominantemente } \\
\text { teóricos }\end{array}$} & $\begin{array}{c}\text { Predominantemente } \\
\text { sobre difusão e/ou } \\
\text { adoção de MTF-I }\end{array}$ & Todas as abordagens & D & $\begin{array}{c}16 \text { (base) } \\
0 \text { (ref) }\end{array}$ \\
\hline & & Todas as temáticas & Todas as abordagens & $E$ & 5 \\
\hline Um & Teóricos e empíricos & Todas as temáticas & Todas as abordagens & $\mathrm{F}$ & 151 \\
\hline
\end{tabular}

Fonte: Elaborado pelos autores. 
- Análise dos artigos: conforme mencionado anteriormente, o presente artigo apresenta o resultado da análise dos estudos qualitativos empíricos relacionados à difusão e adoção de MTF-Is. O objetivo dessa análise é proporcionar uma visão geral dos estudos qualitativos empíricos existentes sobre MTF-Is. Para tanto, utilizou-se o seguinte critério: realizar o levantamento dos dados bibliométricos e a análise do conteúdo dos trabalhos comparando eventualmente os resultados para identificar possíveis divergências ou convergências entre eles, bem como lacunas de pesquisa e recomendações para estudos futuros (ver, por exemplo, Graner \& Mißler -Behr, 20I2). Nesse sentido, os nove dos dez artigos do grupo A foram analisados, tendo em vista que, em um deles, somente o resumo estava disponível. De forma geral, procurou-se identificar, além dos objetivos e resultados dos estudos, como foi empregado o método qualitativo. Assim, os resultados da análise são apresentados na próxima seção.

\section{RESULTADOS}

Com relação ao total de artigos levantados, verificou-se uma predominância de pesquisas relacionadas a um único MTF-I, sejam elas relacionadas ao estudo de um existente ou à proposição de novo. Esses artigos que tratavam de um único MTF-I foram desconsiderados na análise deste estudo. Assim, analisaram-se apenas os artigos que tratavam de mais de um MTF-I, em especial aqueles cujas pesquisas utilizaram a abordagem qualitativa empírica com foco em difusão e/ou adoção de MTF-I (grupo A). Dos artigos que tratavam de mais de um MTF-I com a temática difusão e/ou adoção (grupos A, B e D), verificou-se a existência de mais estudos quantitativos empíricos que abordam difusão e/ou adoção de MTF-I em relação àqueles da mesma temática, mas com abordagem qualitativa empírica. Quando se analisaram esses artigos ao longo do tempo, não se verificou nenhuma tendência entre as pesquisas, conforme mostra o Gráfico I.

Em relação ao número de artigos publicados por autor, de acordo com os critérios estabelecidos para este trabalho, todos os autores tiveram somente um. $\mathrm{O}$ Quadro 2 apresenta os trabalhos com seus referidos autores, suas referências, bem como os países em que residem, as universidades ou organizações das quais fazem parte e a quantidade de citações em cada base de dados utilizada (Scopus e Web of Science), em que ND diz respeito aos artigos que não estão disponíveis na referida base. Destaca-se que a maior parte dos trabalhos foi realizada por pesquisadores europeus (por exemplo, Libutti, 2000; Lichtenthaler, 2005; Scozzi, Garavelli, \& Crowston, 2005; Igartua et al., 2010), e encontraram-se dois estudos de autores brasileiros (Lemos \& Porto, I998; Laurenti, Rozenfeld, \& Franieck, 20I2). 
GRÁFICO I

\section{NÚMERO DE ARTIGOS AO LONGO DOS ANOS}

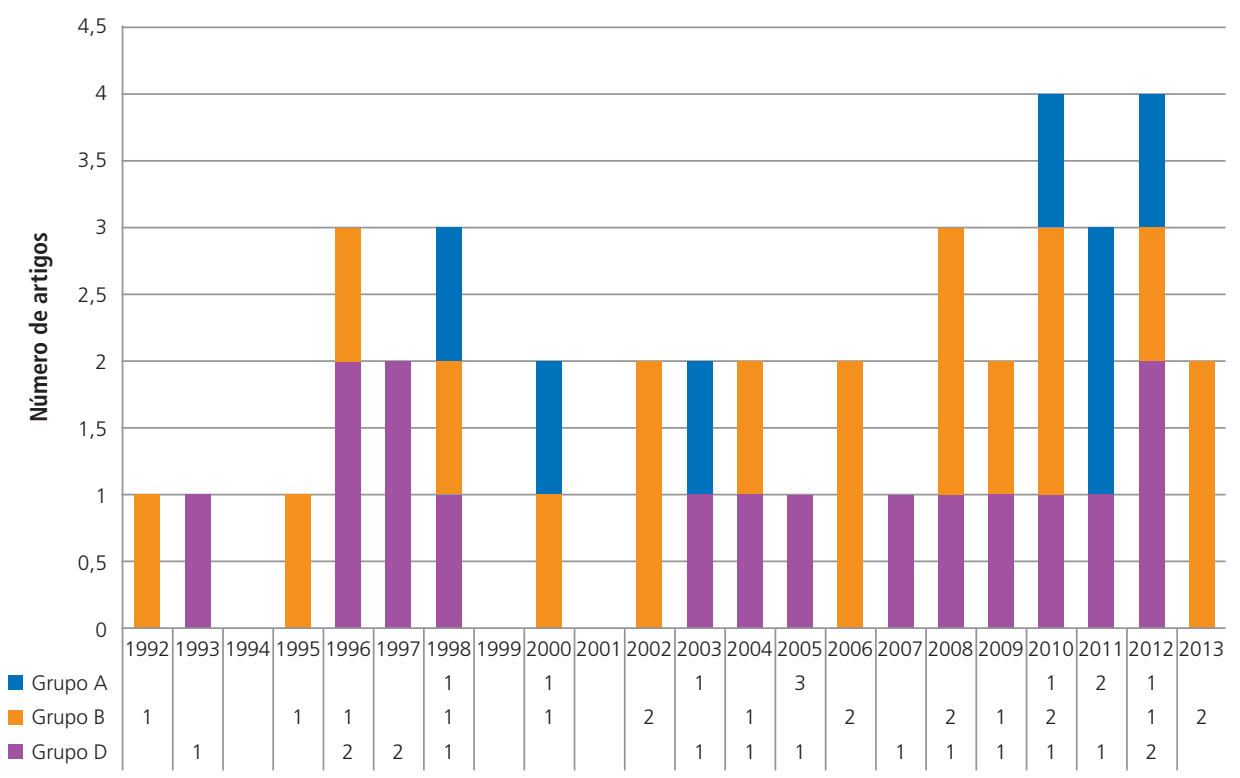

Fonte: Elaborado pelos autores.

\section{QUADRO 2}

\section{DADOS BIBLIOMÉTRICOS}

\begin{tabular}{|c|c|c|c|c|c|c|}
\hline \multirow{2}{*}{ TÍTULO } & \multirow[b]{2}{*}{ AUTOR } & \multirow{2}{*}{ REFERÊNCIA } & \multirow{2}{*}{$\begin{array}{l}\text { PAÍS DOS } \\
\text { AUTORES }\end{array}$} & \multirow{2}{*}{$\begin{array}{l}\text { UNIVERSIDADE/ } \\
\text { ORGANIZAÇÃO } \\
\text { DOS AUTORES }\end{array}$} & \multicolumn{2}{|c|}{ CITAÇÕES } \\
\hline & & & & & SCOPUS & $\begin{array}{l}\text { WEB OF } \\
\text { SCIENCE }\end{array}$ \\
\hline $\begin{array}{l}\text { "Technological } \\
\text { forecasting } \\
\text { techniques and }\end{array}$ & Lemos, A. D. & & & $\begin{array}{l}\text { Universidade } \\
\text { Federal do Rio } \\
\text { Grande do Sul }\end{array}$ & & \\
\hline $\begin{array}{l}\text { competitıve } \\
\text { intelligence: tools } \\
\text { for improving } \\
\text { the innovation } \\
\text { process" }\end{array}$ & Porto, A. C. & $\begin{array}{l}\text { Lemos e Porto } \\
\text { (1998) }\end{array}$ & Brasil & Digitel S. A. & 17 & 14 \\
\hline
\end{tabular}

(continua) 
QUADRO 2 (CONTINUAÇÃO)

DADOS BIBLIOMÉTRICOS

\begin{tabular}{|c|c|c|c|c|c|c|}
\hline \multirow[b]{2}{*}{ TíTULO } & \multirow[b]{2}{*}{ AUTOR } & \multirow[b]{2}{*}{ REFERÊNCIA } & \multirow[b]{2}{*}{$\begin{array}{l}\text { PAÍS DOS } \\
\text { AUTORES }\end{array}$} & \multirow{2}{*}{$\begin{array}{l}\text { UNIVERSIDADE/ } \\
\text { ORGANIZAÇÃO } \\
\text { DOS AUTORES }\end{array}$} & \multicolumn{2}{|c|}{ CITAÇÕES } \\
\hline & & & & & SCOPUS & $\begin{array}{l}\text { WEB OF } \\
\text { SCIENCE }\end{array}$ \\
\hline $\begin{array}{l}\text { "Building } \\
\text { competitive } \\
\text { skills in small } \\
\text { and medium- } \\
\text {-sized enterprises } \\
\text { through innovation } \\
\text { management } \\
\text { techniques: } \\
\text { overview of an } \\
\text { Italian experience' }\end{array}$ & Libutti, L. & Libutti (2000) & Itália & $\begin{array}{l}\text { Institute for } \\
\text { Studies on } \\
\text { Research } \\
\text { and Scientific } \\
\text { Documentation }\end{array}$ & ND & 5 \\
\hline $\begin{array}{l}\text { "Structured } \\
\text { methods } \\
\text { in product } \\
\text { development" }\end{array}$ & $\begin{array}{l}\text { Ghaemmaghami, } \\
\text { S. } \\
\text { Bucciarelli, L. }\end{array}$ & $\begin{array}{l}\text { Ghaemmaghami } \\
\text { e Bucciarelli } \\
\text { (2003) }\end{array}$ & $\begin{array}{l}\text { Estados } \\
\text { Unidos }\end{array}$ & $\begin{array}{l}\text { Massachusetts } \\
\text { Institute of } \\
\text { Technology, } \\
\text { Cambridge }\end{array}$ & ND & 2 \\
\hline $\begin{array}{l}\text { "The choice } \\
\text { of technology } \\
\text { intelligence } \\
\text { methods in } \\
\text { multinationals: } \\
\text { towards a } \\
\text { contingency } \\
\text { approach" }\end{array}$ & Lichtenthaler, E. & $\begin{array}{l}\text { Lichtenthaler } \\
(2005)\end{array}$ & Suíça & $\begin{array}{l}\text { Center for } \\
\text { Enterprise } \\
\text { Science, Group } \\
\text { for Technology } \\
\text { and Innovation } \\
\text { Management, } \\
\text { Swiss Federal } \\
\text { Institute of } \\
\text { Technology } \\
\text { Zurich }\end{array}$ & 32 & 21 \\
\hline $\begin{array}{l}\text { "Methods for } \\
\text { modeling and } \\
\text { supporting } \\
\text { innovation } \\
\text { processes in } \\
\text { SMEs" }\end{array}$ & Scozzi, B. & $\begin{array}{l}\text { Scozzi et al. } \\
(2005)\end{array}$ & $\begin{array}{l}\text { Estados } \\
\text { Unidos }\end{array}$ & $\begin{array}{l}\text { Dipartimento } \\
\text { di Ingegneria } \\
\text { per l'Ambiente } \\
\text { e lo Sviluppo } \\
\text { Sostenibile, } \\
\text { Politecnico di } \\
\text { Bari } \\
\text { School of } \\
\text { Information } \\
\text { Studies, } \\
\text { Syracuse } \\
\text { University }\end{array}$ & 40 & ND \\
\hline
\end{tabular}

(continua) 
QUADRo 2 (CONTINUAÇÃo)

DADOS BIBLIOMETTRICOS

\begin{tabular}{|c|c|c|c|c|c|c|}
\hline \multirow[b]{2}{*}{ TÍTULO } & \multirow[b]{2}{*}{ AUTOR } & \multirow[b]{2}{*}{ REFERÊNCIA } & \multirow{2}{*}{$\begin{array}{l}\text { PAÍS DOS } \\
\text { AUTORES }\end{array}$} & \multirow{2}{*}{$\begin{array}{l}\text { UNIVERSIDADE/ } \\
\text { ORGANIZAÇÃO } \\
\text { DOS AUTORES }\end{array}$} & \multicolumn{2}{|c|}{ CITAÇÕES } \\
\hline & & & & & SCOPUS & $\begin{array}{l}\text { WEB OF } \\
\text { SCIENCE }\end{array}$ \\
\hline \multirow{5}{*}{$\begin{array}{l}\text { "An exploratory } \\
\text { study of the use } \\
\text { of quality tools } \\
\text { and techniques } \\
\text { in product } \\
\text { development" }\end{array}$} & Thia, C. W. & \multirow{5}{*}{ Thia et al. (2005) } & \multirow{5}{*}{ Cingapura } & $\begin{array}{l}\text { Department } \\
\text { of Mechanical } \\
\text { Engineering, } \\
\text { National } \\
\text { University of } \\
\text { Singapore }\end{array}$ & \multirow{5}{*}{19} & \multirow{5}{*}{ ND } \\
\hline & Chai, K. H. & & & \multirow{4}{*}{$\begin{array}{l}\text { Department } \\
\text { of Industrial } \\
\text { and Systems } \\
\text { Engineering, } \\
\text { National } \\
\text { University of } \\
\text { Singapore }\end{array}$} & & \\
\hline & Bauly, J. & & & & & \\
\hline & & & & & & \\
\hline & Xin, $Y$. & & & & & \\
\hline \multirow{3}{*}{$\begin{array}{l}\text { "How innovation } \\
\text { management } \\
\text { techniques } \\
\text { support an } \\
\text { open innovation } \\
\text { strategy" }\end{array}$} & Igartua, J. I. & \multirow{3}{*}{$\begin{array}{l}\text { Igartua et al. } \\
\text { (2010) }\end{array}$} & \multirow{3}{*}{ Espanha } & $\begin{array}{l}\text { Mondragon } \\
\text { University }\end{array}$ & \multirow{3}{*}{7} & \multirow{3}{*}{ ND } \\
\hline & Garrigós, J. A. & & & Universidad & & \\
\hline & $\begin{array}{l}\text { Hervas-Oliver, } \\
\text { J. L. }\end{array}$ & & & $\begin{array}{l}\text { Politécnica de } \\
\text { Valencia }\end{array}$ & & \\
\hline \multirow{2}{*}{$\begin{array}{l}\text { "Maximizing } \\
\text { product innovation } \\
\text { through adaptive } \\
\text { application of user- } \\
\text {-centered methods } \\
\text { for defining } \\
\text { customer value" }\end{array}$} & Olsen, T. O. & \multirow[b]{2}{*}{$\begin{array}{l}\text { Olsen e Welo } \\
\text { (2011) }\end{array}$} & \multirow[b]{2}{*}{ Noruega } & $\begin{array}{l}\text { Department } \\
\text { of Engineering } \\
\text { Design and }\end{array}$ & & \\
\hline & Welo, T. & & & $\begin{array}{l}\text { Norwegian } \\
\text { University of } \\
\text { Science and } \\
\text { Technology }\end{array}$ & 2 & ND \\
\hline \multirow{3}{*}{$\begin{array}{l}\text { "A process for } \\
\text { configuring } \\
\text { technology } \\
\text { management } \\
\text { tools" }\end{array}$} & Keltsch, J. & \multirow{3}{*}{$\begin{array}{l}\text { Keltsch et al. } \\
\text { (2011) }\end{array}$} & \multirow{3}{*}{ Inglaterra } & \multirow{3}{*}{$\begin{array}{l}\text { University of } \\
\text { Cambridge }\end{array}$} & \multirow{3}{*}{1} & \multirow{3}{*}{ ND } \\
\hline & Probert, D. & & & & & \\
\hline & Phaal, R. & & & & & \\
\hline
\end{tabular}

(continua) 


\section{QUADRO 2 (CONCLUSÃO)}

\section{DADOS BIBLIOMÉTRICOS}

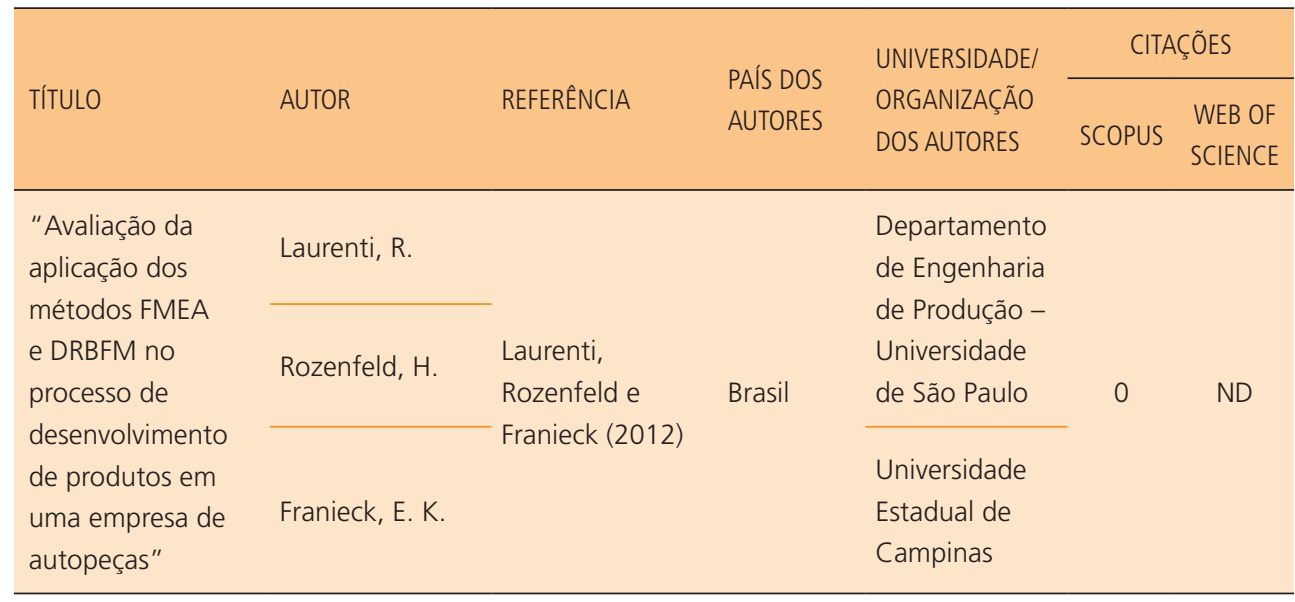

Fonte: Elaborado pelos autores.

Destacam-se alguns autores que tiveram outras publicações acerca do tema MTF-I. O Quadro 3 apresenta os dados de outras publicações encontradas nos grupos B e D dos autores analisados neste trabalho. Entretanto, esses artigos não foram unidades de análise, pois não cumpriram os critérios do grupo $\mathrm{A}$, foco deste trabalho.

\section{QUADRO 3}

AUTORES QUE PUBLICARAM EM OUTROS GRUPOS (GRUPOS B E D)

\begin{tabular}{|c|c|c|c|}
\hline AUTOR & REFERÊNCIA & TíTULO & GRUPO \\
\hline Chai, K. H. & \multirow{2}{*}{ Chai e Xin (2006) } & \multirow{2}{*}{$\begin{array}{l}\text { "The application of new product development tools } \\
\text { in industry: the case of Singapore" }\end{array}$} & \multirow{2}{*}{ B } \\
\hline Xin, $Y$. & & & \\
\hline Probert, D. & Brady et al. (1997) & $\begin{array}{l}\text { "Tools for technology management: an academic } \\
\text { perspective" }\end{array}$ & D \\
\hline Phaal, R. & $\begin{array}{l}\text { Lee, Mortara, Kerr, Phaal } \\
\text { e Probert (2012) }\end{array}$ & $\begin{array}{l}\text { "Analysis of document-mining techniques and tools } \\
\text { for technology intelligence: discovering knowledge } \\
\text { from technical documents" }\end{array}$ & D \\
\hline
\end{tabular}

Fonte: Elaborado pelos autores. 
Com relação aos periódicos que mais publicaram artigos oriundos de pesquisas qualitativas empíricas sobre MTF-I, não houve algum com mais de uma publicação. Já com relação às palavras-chave utilizadas para descrever os artigos, verificou-se que a maioria daquelas mais citadas está relacionada aos ambientes industriais e tecnológicos. As palavras mais frequentes foram: strategic planning (quatro artigos), technological forecasting (quatro), industrial management (quatro), competition (três), competitive intelligence (dois), innovation (dois), quality assurance (dois), competitive intelligence (dois), managers (dois), methods (dois) e research and development management (dois). Outras palavras utilizadas pelos artigos analisados tiveram somente uma citação. A partir dessa frequência de palavras-chave, percebe-se que a maior parte dos estudos foi realizada em ambientes industriais.

\subsection{ANÁLISE DO CONTEÚdO DOS ARTIGOS LEVANTADOS}

Após a análise dos dados bibliométricos dos dez artigos levantados, realizou-se uma análise individual dos trabalhos para aprofundar o conteúdo e auxiliar na construção de um panorama geral das pesquisas acerca de MTF-I com abordagem qualitativa empírica (grupo A). Nesse sentido, o Quadro 4 (organizado por ano de publicação dos trabalhos) apresenta as temáticas abordadas em cada um, bem como os objetivos de cada artigo. Os artigos são classificados em "adoção" e "difusão". Dessa forma, oito dos nove artigos analisados possuem ênfase na adoção de MTF-Is, assim como o resumo analisado.

\section{QUADRO 4}

\section{DADOS GERAIS DOS ARTIGOS LEVANTADOS}

\begin{tabular}{|c|c|c|}
\hline REFERÊNCIA & GRUPO & OBJETIVO \\
\hline $\begin{array}{l}\text { Lemos e Porto } \\
\text { (1998) }\end{array}$ & Adoção & $\begin{array}{l}\text { Analisar como uma empresa brasileira da indústria eletrônica, } \\
\text { que produz modems e multiplexadores para os mercados } \\
\text { internos e globais, lida com MTF-I como forma de melhorar o } \\
\text { seu processo de inovação para alcançar vantagem competitiva. }\end{array}$ \\
\hline Libutti (2000) & Adoção & $\begin{array}{l}\text { Reportar o trabalho e os resultados do projeto Técnicas de } \\
\text { Gestão da Inovação para Clusters Industriais (Innovation } \\
\text { Management Techniques for Industry Cluters - Imtic), apoiado } \\
\text { pelo Programa de Inovação da Comissão Europeia. A finalidade } \\
\text { do projeto era fazer com que pequenas e médias empresas } \\
\text { tenham consciência das possibilidades oferecidas pelos MTF-I } \\
\text { no planejamento e na execução de estratégias de negócios de } \\
\text { longo prazo. }\end{array}$ \\
\hline
\end{tabular}

(continua) 


\section{QUADRO 4 (CONCLUSÃO)}

\section{DADOS GERAIS DOS ARTIGOS LEVANTADOS}

\begin{tabular}{|c|c|c|}
\hline REFERÊNCIA & GRUPO & OBJETIVO \\
\hline $\begin{array}{l}\text { Ghaemmaghami } \\
\text { e Bucciarelli } \\
\text { (2003) }\end{array}$ & Adoção & $\begin{array}{l}\text { Verificar como os MTF-I são desenvolvidos para gerenciar o } \\
\text { desenho dos produtos e processos de desenvolvimento. }\end{array}$ \\
\hline $\begin{array}{l}\text { Lichtenthaler } \\
\text { (2005) }\end{array}$ & Adoção & Fornecer insights sobre a escolha de MTF-I por multinacionais. \\
\hline $\begin{array}{l}\text { Scozzi et al. } \\
\text { (2005) }\end{array}$ & Difusão & $\begin{array}{l}\text { Investigar os MTF-Is que podem ser usados para apoiar e } \\
\text { melhorar os processos de inovação nas pequenas e médias } \\
\text { empresas. }\end{array}$ \\
\hline Thia et al. (2005) & Adoção & $\begin{array}{l}\text { Investigar as razões para a adoção ou não de MTF-I na } \\
\text { indústria. }\end{array}$ \\
\hline $\begin{array}{l}\text { lgartua et al. } \\
\text { (2010) }\end{array}$ & Adoção & $\begin{array}{l}\text { Analisar a utilização de MTF-I no setor de manufatura de } \\
\text { elevadores da Espanha. }\end{array}$ \\
\hline $\begin{array}{l}\text { Olsen e Welo } \\
\text { (2011) }\end{array}$ & Adoção & $\begin{array}{l}\text { Identificar os MTF-Is existentes utilizados para aumentar a } \\
\text { compreensão do cliente no processo de inovação. Aplicar } \\
\text { uma pequena seleção dos MTF-I em um estudo de caso } \\
\text { para aumentar a compreensão da sua aplicabilidade e para } \\
\text { fornecer orientações para as suas aplicações em projetos de } \\
\text { desenvolvimento de produtos posteriores. }\end{array}$ \\
\hline $\begin{array}{l}\text { Keltsch et al. } \\
(2011)\end{array}$ & Adoção & $\begin{array}{l}\text { Analisar resultados das melhores práticas de casos da indústria e } \\
\text { sugerir atividades para cada fase do processo de implementação } \\
\text { de MTF-I dentro de suas organizações. }\end{array}$ \\
\hline $\begin{array}{l}\text { Laurenti et al. } \\
\text { (2012) }\end{array}$ & Adoção & $\begin{array}{l}\text { Apresentar os resultados de um estudo de caso realizado } \\
\text { para avaliar a aplicação dos métodos de análise do modo e } \\
\text { dos efeitos de falha (failure mode and effect analysis - FMEA) } \\
\text { e revisão de projeto baseada nos modos de falha (design } \\
\text { review based on failure mode - DRBFM) no processo de } \\
\text { desenvolvimento de produtos de uma empresa multinacional } \\
\text { do setor automotivo. }\end{array}$ \\
\hline
\end{tabular}

Fonte: Elaborado pelos autores.

O Quadro 5 apresenta os trabalhos e as respectivas estratégias de investigação e técnicas de coleta de dados, bem como o contexto no qual foram pesquisados. 
Em relação às estratégias de investigação, observou-se a predominância da utilização de estudos de caso, seguido de estudos multicaso e uma utilização da pesquisa-ação. Além disso, percebeu-se uma variedade de técnicas de coleta de dados nos trabalhos analisados.

QUADRO 5

TÉCNICAS DE COLETA DE DADOS E CONTEXTOS DAS PESQUISAS DOS ARTIGOS LEVANTADOS

\begin{tabular}{|c|c|c|c|}
\hline REFERÊNCIA & $\begin{array}{l}\text { ESTRATÉGIA DE } \\
\text { INVESTIGAÇÃOO }\end{array}$ & $\begin{array}{l}\text { TÉCNICA DE COLETA } \\
\text { DE DADOS }\end{array}$ & CONTEXTO \\
\hline $\begin{array}{l}\text { Lemos e Porto } \\
\text { (1998) }\end{array}$ & $\begin{array}{l}\text { Estudo de } \\
\text { caso }\end{array}$ & $\begin{array}{l}\text { Pesquisa } \\
\text { documental }\end{array}$ & $\begin{array}{l}\text { Empresa brasileira de médio porte } \\
\text { pertencente à indústria eletrônica. }\end{array}$ \\
\hline Libutti (2000) & Pesquisa-ação & $\begin{array}{l}\text { Entrevistas } \\
\text { baseadas em } \\
\text { questionários e } \\
\text { pesquisas on-line } \\
\text { em todos os } \\
\text { meios eletrônicos } \\
\text { disponíveis }\end{array}$ & $\begin{array}{l}\text { Clusters industriais em cinco regiões } \\
\text { italianas, nas áreas de: } 1 \text {. inovação } \\
\text { em, 2. technology watch, 3. pesquisa } \\
\text { tecnológica, 4. gerenciamento de } \\
\text { direitos de propriedade intelectual e } \\
\text { 5. gestão da qualidade. }\end{array}$ \\
\hline $\begin{array}{l}\text { Ghaemmaghami } \\
\text { e Bucciarelli } \\
\text { (2003) }\end{array}$ & $\begin{array}{l}\text { Estudo de } \\
\text { caso }\end{array}$ & $\begin{array}{l}\text { Observação } \\
\text { empírica, } \\
\text { etnografia }\end{array}$ & $\begin{array}{l}\text { Empresa de médio porte localizada } \\
\text { na Costa Leste dos Estados Unidos. }\end{array}$ \\
\hline $\begin{array}{l}\text { Lichtenthaler } \\
\text { (2005) }\end{array}$ & $\begin{array}{l}\text { Estudo } \\
\text { multicaso }\end{array}$ & Entrevistas & $\begin{array}{l}\text { Vinte e cinco principais empresas } \\
\text { europeias e norte-americanas } \\
\text { na indústria farmacêutica, } \\
\text { equipamentos de telecomunicações } \\
\text { e as indústrias de automóvel/ } \\
\text { máquinas. }\end{array}$ \\
\hline $\begin{array}{l}\text { Scozzi et al. } \\
(2005)\end{array}$ & $\begin{array}{l}\text { Estudo } \\
\text { multicaso }\end{array}$ & $\begin{array}{l}\text { Entrevistas } \\
\text { baseadas em } \\
\text { questionários }\end{array}$ & $\begin{array}{l}\text { Dezenove pequenas e médias } \\
\text { empresas dos setores têxtil, } \\
\text { alimentício, mecânico, agrícola e } \\
\text { moveleiro. }\end{array}$ \\
\hline
\end{tabular}


QUADRO 5 (CONCLUSÃO)

TÉCNICAS DE COLETA DE DADOS E CONTEXTOS DAS PESQUISAS DOS ARTIGOS LEVANTADOS

\begin{tabular}{|c|c|c|c|}
\hline REFERÊNCIA & $\begin{array}{l}\text { ESTRATÉGIA DE } \\
\text { INVESTIGAÇÃO }\end{array}$ & $\begin{array}{l}\text { TÉCNICA DE COLETA } \\
\text { DE DADOS }\end{array}$ & CONTEXTO \\
\hline Thia et al. (2005) & $\begin{array}{l}\text { Estudo } \\
\text { multicaso }\end{array}$ & $\begin{array}{l}\text { Entrevistas } \\
\text { semiestruturadas }\end{array}$ & $\begin{array}{l}\text { Sete empresas que produzem } \\
\text { produtos de consumo, como } \\
\text { aparelhos de televisão, equipamentos } \\
\text { de áudio para outras empresas que } \\
\text { fabricam produtos industriais, tais } \\
\text { como módulo de interface de rede } \\
\text { e disjuntores. Além disso, foram } \\
\text { realizadas entrevistas com três } \\
\text { acadêmicos. }\end{array}$ \\
\hline $\begin{array}{l}\text { lgartua et al. } \\
(2010)\end{array}$ & $\begin{array}{l}\text { Estudo de } \\
\text { caso }\end{array}$ & $\begin{array}{l}\text { Technology } \\
\text { watch, } \\
\text { roadmapping e } \\
\text { planning horizon }\end{array}$ & $\begin{array}{l}\text { Empresa líder no setor de fabricação } \\
\text { e prestadoras de serviços de } \\
\text { manutenção, reparação e instalação } \\
\text { de elevadores, escadas rolantes e } \\
\text { esteiras rolantes da Espanha. }\end{array}$ \\
\hline $\begin{array}{l}\text { Olsen e Welo } \\
\text { (2011) }\end{array}$ & $\begin{array}{l}\text { Estudo de } \\
\text { caso }\end{array}$ & $\begin{array}{l}\text { Levantamento } \\
\text { baseado na } \\
\text { web, entrevistas, } \\
\text { observação e } \\
\text { oficinas }\end{array}$ & $\begin{array}{l}\text { Empresa de desenvolvimento de } \\
\text { cadeiras de escritório. }\end{array}$ \\
\hline $\begin{array}{l}\text { Keltsch et al. } \\
\text { (2011) }\end{array}$ & $\begin{array}{l}\text { Estudo } \\
\text { multicaso }\end{array}$ & $\begin{array}{l}\text { Observação } \\
\text { participante }\end{array}$ & $\begin{array}{l}\text { Treze empresas de diversos setores } \\
\text { da indústria. }\end{array}$ \\
\hline $\begin{array}{l}\text { Laurenti et al. } \\
\text { (2012) }\end{array}$ & $\begin{array}{l}\text { Estudo de } \\
\text { caso }\end{array}$ & Entrevistas & $\begin{array}{l}\text { Empresa de excelência mundial no } \\
\text { desenvolvimento de produtos no } \\
\text { setor automotivo. Considerada um } \\
\text { benchmarking na aplicação da FMEA } \\
\text { com a DRBFM. }\end{array}$ \\
\hline
\end{tabular}

Fonte: Elaborado pelos autores.

O trabalho de Lemos e Porto (I998) aborda os MTF-Is de previsão tecnológica e inteligência competitiva para melhorar o processo de inovação. Como MTF-Is de previsão tecnológica, os autores citam o método de consenso (painel de especialistas que debatem o assunto face a face, ou seja, é um procedimento intuitivo); O método Delphi (painel de especialistas que respondem a várias rodadas de 
questionários, mas que, em geral, não se encontram face a face; trata-se também de um processo intuitivo); modelos estruturais (procedimento mecanicista que tenta desenvolver modelos matemáticos ou analíticos para a realização da previsão); cenários (não é uma técnica formal, mas serve como um guia no auxílio das previsões tentando identificar ameaças e oportunidades para as empresas; trata-se de um procedimento combinado que utiliza abordagens intuitivas, cognitivas e mecanicistas); e vigília tecnológica (um conjunto relacionado de produtos, processos ou procedimentos que visam à administração do fluxo de informação científica, técnica e tecnológica, a fim de auxiliar o processo de inovação dentro das empresas).

Com relação aos MTF-Is de inteligência competitiva, Lemos e Porto (I998) citam: análise estrutural de indústrias, benchmarking, análise de portfólio, engenharia reversa, análise de pontos fortes e fracos de uma situação, cenários, fatores críticos de sucesso e análise da cadeia de valor. Além disso, existem alguns fatores que fazem a ligação entre os MTF-Is de previsão tecnológica e os de inteligência competitiva. Esses fatores, segundo Lemos e Porto (I998), são: futuro, incerteza, informações, tomada de decisão, escolhas estratégicas, inovação e vantagem competitiva. Como resultado do estudo de caso na Digitel S.A., verificou-se que foram utilizadas algumas características dos MTF-Is no desenvolvimento de um produto inovador, o qual gerou vantagem competitiva para a empresa, especialmente no mercado norte-americano. No entanto, é necessário destacar que essa vantagem competitiva alcançada é baseada principalmente em informações inteligentes, ou seja, à informação pública adicionaram-se os conhecimentos e as habilidades dos membros de sua equipe, que são os verdadeiros responsáveis pela vantagem competitiva das empresas.

Libutti (2000), a partir de uma pesquisa-ação realizada na Itália, buscou definir como construir habilidades competitivas em pequenas e médias empresas por meio de MTF-I. O projeto Imtic é uma das ações de sensibilização que visam mostrar para as pequenas e médias empresas as oportunidades de desenvolvimento oferecidas pelos MTF-Is. Estes lidam com a estratégia geral, na medida em que ajudam a formular estratégias dentro de seus contextos. Além disso, criam uma visão holística da inovação e permitem que as empresas aproveitem o máximo das novas tecnologias. Dessa forma, o primeiro passo do projeto foi identificar cinco MTF-Is de potencial interesse para as empresas: vigilância tecnológica, pesquisa tecnológica, inovação em marketing, gestão dos direitos de propriedade intelectual e gestão da qualidade (por exemplo, QFD, FMEA, HACCP ${ }^{\mathrm{I}}$ ). Em seguida, o projeto visou criar uma metodologia para ser utilizada pelos consultores responsáveis pela sua aplicação dentro das empresas e, posteriormente, 
propor os MTF-Is para os clusters industriais individuais selecionados em uma série de reuniões. Com isso, o estudo demonstrou a vontade das empresas participantes em usar MTF-Is úteis para a introdução de processos inovadores e aceitar pessoas de fora da empresa, tendo em vista que o projeto foi implementado por consultores. Os consultores consideraram importantes os seguintes MTF-Is introduzidos nos clusters industriais: vigilância tecnológica e pesquisa tecnológica. As empresas participantes não haviam selecionado o primeiro, já o segundo foi opção de $45 \%$ delas. Os outros MTF-Is escolhidos durante a pesquisa foram FMEA (10\%), HACCP (30\%) e inovação em marketing (15\%).

$\mathrm{O}$ artigo de Ghaemmaghami e Bucciarelli (2003) trata de MTF-I no desenvolvimento de produtos. Os autores utilizaram a perspectiva de que o desenvolvimento de produtos é uma atividade social, ou seja, é o resultado do trabalho humano organizado a fim de transformar um conjunto de requisitos em realidade. $\mathrm{O}$ foco do trabalho está nos MTF-Is desenvolvidos dentro da equipe de projetos de engenharia com o objetivo de gerenciar o design do projeto e o processo de desenvolvimento. A metodologia utilizada para o desenvolvimento de produto na empresa estudada é mostrada na Figura I.

\section{FIGURA I}

\section{METODOLOGIA DE DESENVOLVIMENTO DE PRODUTOS DO} GRUPO DE TRABALHO CRIATIVO DE UMA MÉDIA EMPRESA

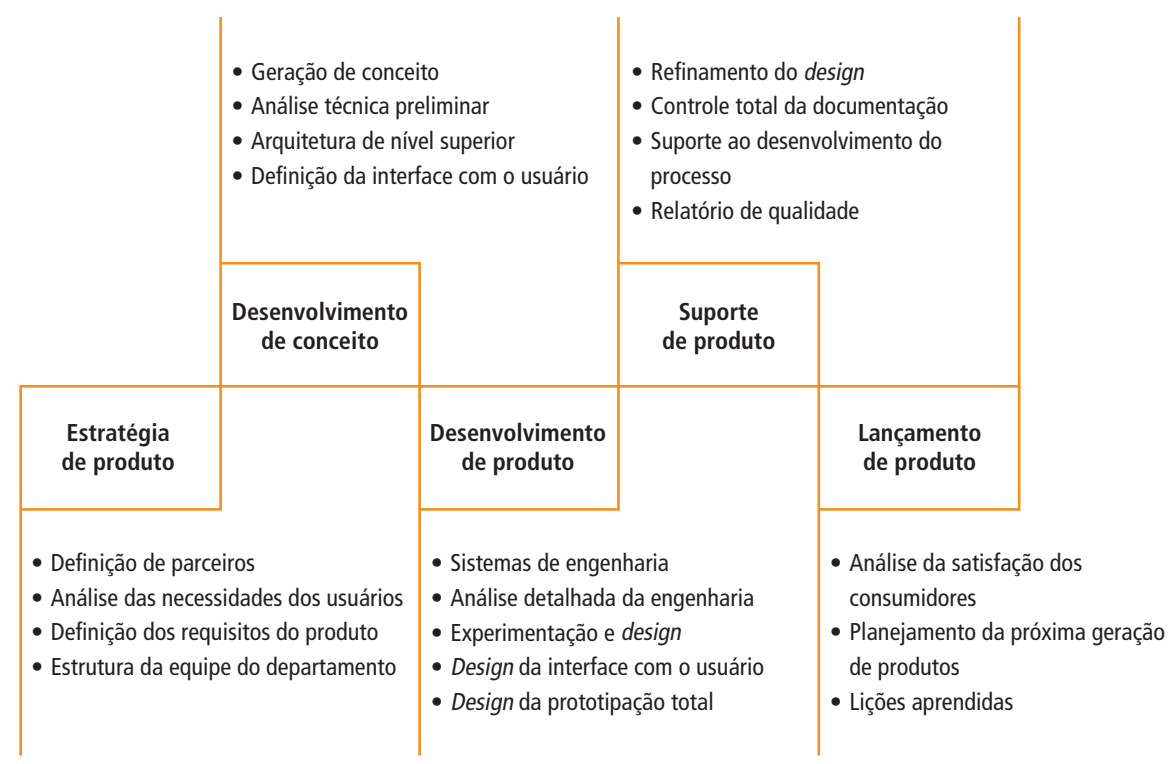

Fonte: Adaptada de Ghaemmaghami e Bucciarelli (2003, p. I35). 
Os resultados mostraram como os MTF-Is são usados, modelados e moldados por participantes de diversas maneiras, com muitos benefícios tangíveis e intangíveis. Essa teoria é apoiada pela observação do design, o que indica que os designers resolvem novos problemas generalizando a partir de um problema semelhante ou reformulando o problema para se adaptar às soluções parciais que já estão disponíveis para eles.

O trabalho de Lichtenthaler (2005) visa auxiliar a escolha de MTF-I em multinacionais. Em seu estudo multicaso, o autor identificou diversos MTF-Is e suas formas de avaliação, ou seja, individuais ou em grupo. Além disso, foram identificadas as funções de geração de informações e de aprendizagem dos MTF-Is. A análise realizada por Lichtenthaler (2005) é mostrada no Quadro 6.

QUADRO 6

POSSIBILIDADES DE AVALIAÇÃO E FUNÇŐES DOS MTF-IS

\begin{tabular}{|c|c|c|c|c|c|c|c|}
\hline \multirow{2}{*}{ MTF-Is } & \multirow{2}{*}{$\begin{array}{l}\text { AVALIAÇÃO } \\
\text { INDIVIDUAL }\end{array}$} & \multirow{2}{*}{$\begin{array}{l}\text { AVALIAÇÃO } \\
\text { EM GRUPO }\end{array}$} & \multicolumn{3}{|c|}{ GERAÇÃO DE INFORMAÇÃO } & \multicolumn{2}{|c|}{ APRENDIZAGEM } \\
\hline & & & EXTRAPOLATIVA & EXPLORATÓRIA & NORMATIVA & ORGANIZACIONAL & INDIVIDUAL \\
\hline $\begin{array}{l}\text { Análise de } \\
\text { frequência de } \\
\text { publicação }\end{array}$ & $x$ & & $x$ & & & & $x$ \\
\hline $\begin{array}{l}\text { Análise de } \\
\text { citações } \\
\text { publicadas }\end{array}$ & $x$ & & $x$ & & & & $x$ \\
\hline $\begin{array}{l}\text { Análise de } \\
\text { conferência } \\
\text { quantitativa }\end{array}$ & $x$ & & $x$ & & & & $x$ \\
\hline $\begin{array}{l}\text { Análise de } \\
\text { frequência de } \\
\text { patente }\end{array}$ & $x$ & & $x$ & & & & $x$ \\
\hline $\begin{array}{l}\text { Análise de } \\
\text { citações de } \\
\text { patente }\end{array}$ & $x$ & & $x$ & $x$ & & & $x$ \\
\hline $\begin{array}{l}\text { Análise de } \\
\text { curva-S }\end{array}$ & $x$ & $x$ & $x$ & & $x$ & $x$ & $x$ \\
\hline $\begin{array}{l}\text { Estudos de } \\
\text { benchmarking }\end{array}$ & $x$ & $x$ & $x$ & & & $x$ & $x$ \\
\hline Portfólios & $x$ & $x$ & $x$ & & $x$ & $x$ & $x$ \\
\hline
\end{tabular}




\section{QUADRO 6 (CONCLUSÃO)}

POSSIBILIDADES DE AVALIAÇÃO E FUNÇÕES DOS MTF-I

\begin{tabular}{|c|c|c|c|c|c|c|c|}
\hline \multirow{2}{*}{ MTF-Is } & \multirow{2}{*}{$\begin{array}{l}\text { AVALIAÇÃO } \\
\text { INDIVIDUAL }\end{array}$} & \multirow{2}{*}{$\begin{array}{l}\text { AVALIAÇÃO } \\
\text { EM GRUPO }\end{array}$} & \multicolumn{3}{|c|}{ GERAÇÃO DE INFORMAÇÃO } & \multicolumn{2}{|c|}{ APRENDIZAGEM } \\
\hline & & & EXTRAPOLATIVA & EXPLORATÓRIA & NORMATIVA & ORGANIZACIONAL & INDIVIDUAL \\
\hline Estudos Delphi & $x$ & & $x$ & $x$ & & & $x$ \\
\hline $\begin{array}{l}\text { Painéis de } \\
\text { especialistas }\end{array}$ & $x$ & $x$ & $x$ & $x$ & & $x$ & $x$ \\
\hline $\begin{array}{l}\text { Entrevistas com } \\
\text { especialistas } \\
\text { flexíveis }\end{array}$ & $x$ & & $x$ & $x$ & & & $x$ \\
\hline $\begin{array}{l}\text { Roadmaps } \\
\text { tecnológicos }\end{array}$ & $x$ & $x$ & $x$ & & & $x$ & $x$ \\
\hline $\begin{array}{l}\text { Roadmaps } \\
\text { de produtos } \\
\text { tecnológicos }\end{array}$ & $x$ & $x$ & $x$ & & & $x$ & $x$ \\
\hline $\begin{array}{l}\text { Roadmaps de } \\
\text { produtos }\end{array}$ & $x$ & $x$ & $x$ & & & $x$ & $x$ \\
\hline $\begin{array}{l}\text { Curvas de } \\
\text { experiência }\end{array}$ & $x$ & $x$ & $x$ & & $x$ & $x$ & $x$ \\
\hline Simulações & $x$ & & & $x$ & & & $x$ \\
\hline $\begin{array}{l}\text { Modelos de } \\
\text { opções de } \\
\text { precificação }\end{array}$ & $x$ & $x$ & & $x$ & & $x$ & $x$ \\
\hline $\begin{array}{l}\text { Análise de } \\
\text { cenários }\end{array}$ & $x$ & $x$ & & $x$ & $x$ & $x$ & $x$ \\
\hline $\begin{array}{l}\text { Análise lead } \\
\text { user }\end{array}$ & $x$ & $x$ & $x$ & $x$ & & $x$ & $x$ \\
\hline $\begin{array}{l}\text { Desdobramento } \\
\text { da função } \\
\text { qualidade }\end{array}$ & $x$ & $x$ & $x$ & & & $x$ & $x$ \\
\hline
\end{tabular}

Fonte: Elaborado pelos autores com base em Lichtenthaler (2005).

Com relação à função de geração de informações orientada para o futuro, há três formas citadas por Lichtenthaler (2005): extrapolativa, exploratória e normativa. A forma extrapolativa traduz os desenvolvimentos antigos e atuais 
para o futuro e tenta desenvolver a imagem mais provável do futuro. A geração de informação exploratória, ao contrário, tem por objetivo identificar possíveis desenvolvimentos futuros e desenvolver diferentes imagens possíveis do futuro. Já a geração de informações normativa visa analisar uma imagem prevista para o futuro e identificar possíveis caminhos que levam a essa imagem. Os processos de aprendizagem podem ser divididos em individual e organizacional. Quanto mais pessoas participarem do processo de aprendizagem, maiores serão as chances de elas atingirem a aprendizagem organizacional, e esse é o objetivo principal. Nesse processo, devem-se utilizar as avaliações em grupo; caso contrário, podem-se adotar as individuais.

Dessa forma, em uma avaliação de uma situação específica, as empresas precisam escolher um MTF-I e a forma de avaliação apropriados. Nesse sentido, essas escolhas, segundo o estudo realizado por Lichtenthaler (2005), são influenciadas por diversos fatores identificados na Figura 2.

\section{FIGURA 2}

\section{A ESCOLHA DOS MTF-IS E DAS FORMAS DE AVALIAÇÃO E SEUS FATORES DE CONTINGÊNCIA}

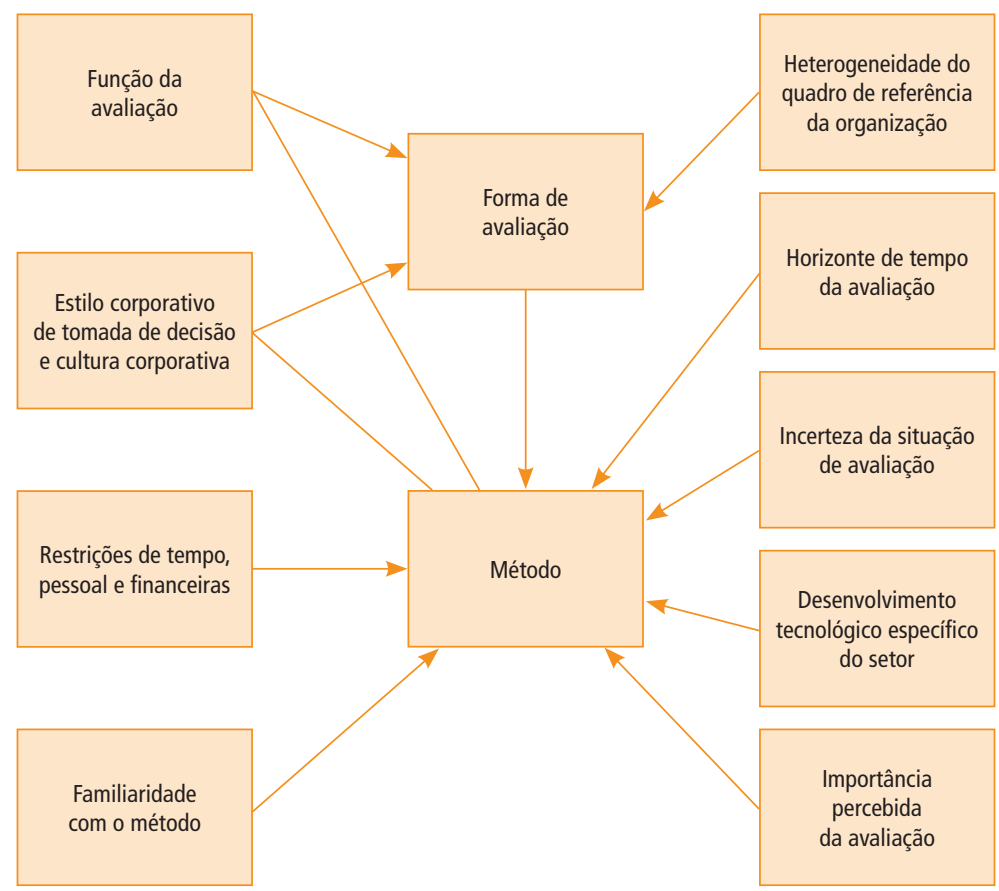

Fonte: Adaptada de Lichtenthaler (2005, p. 393). 
Como resultado desse estudo, as empresas estudadas utilizam os MTF-I mais complexos e caros, em contraste com pesquisas passadas. Além disso, cada MTF-I possui uma relevância particular para cada empresa. A partir dessa análise, os conjuntos de MTF-Is adequados para as empresas devem ser determinados levando em consideração as diferenças entre indústrias e outros fatores de contingência identificados nesse estudo.

Scozzi et al. (2005) trabalham os MTF-Is para a modelagem da inovação e o apoio a ela em processos, em pequenas e médias empresas. Para isso, os autores buscam insights sobre os processos de inovação a partir de sete perspectivas para análise. Essas perspectivas e seus MTF-Is são mostrados no Quadro 7. Além disso, os autores abordam MTF-Is que suportam o processo de inovação. Dessa forma, ao adotarem as sete perspectivas de análise para o desenvolvimento do processo de inovação, os autores utilizaram modelos para planejamento, desenvolvimento e aprendizagem.

\section{QUADRO 7}

\section{PERSPECTIVAS DE ANÁLISE E SEUS MTF-IS}

\begin{tabular}{ll}
\hline PERSPECTIVAS DE ANÁLISE & MTF-IS CITADOS \\
\hline Sequência de tarefas & Fluxogramas, IDEFO* e IDEF3** \\
\hline $\begin{array}{l}\text { Decisões que evoluem ao longo } \\
\text { do tempo }\end{array}$ & $\begin{array}{l}\text { Simulações, árvores de decisões, lógica projetada, } \\
\text { processo de hierarquia analítica, Petri net e diagramas } \\
\text { de transição }\end{array}$ \\
\hline Processo estratégico & Fatores críticos de sucesso e mapas \\
\hline Processo político & $\begin{array}{l}\text { Mapas cognitivos, modelos de fluxo de trabalhos ativos } \\
\text { e modelagem de interação de discurso }\end{array}$ \\
\hline Processo interpretativo & Mapas cognitivos e IDEF5 \\
\hline Processo criativo & $\begin{array}{l}\text { Brainstorming, diagrama de afinidade, método Delphi } \\
\text { e método "pensar fora da caixa" }\end{array}$ \\
\hline Fluxo de comunicação e informação & \begin{tabular}{l} 
Fluxograma, IDEF1 e diagramas de papéis ativos \\
\hline
\end{tabular}
\end{tabular}

* IDEFo - Icam DEFinition for Function Modeling.

$* * \mathrm{IDEF}_{3}$ - Integrated DEFinition for Process Description Capture Method.

Fonte: Elaborado pelos autores com base em Scozzi et al. (2005).

O principal resultado do trabalho de Scozzi et al. (2005) foi a identificação dos problemas que enfrentam as pequenas e médias empresas no processo de inovação e o possível apoio oferecido pelos MTF-Is. 
Thia et al. (2005) realizaram um estudo exploratório sobre o uso de MTF-I no desenvolvimento de produtos em sete empresas, cujo objetivo foi investigar as razões para a adoção ou não de MTF-I na indústria. A partir desse estudo multicaso, Thia et al. (2005) identificaram o número de empresas que conhecem os MTF-Is e quantas efetivamente os adotam. O Quadro 8 apresenta essa relação.

\section{QUADRO 8}

\section{EMPRESAS QUE CONHECEM E ADOTAM OS MTF-IS}

\begin{tabular}{lccc}
\hline MTF-IS & $\begin{array}{c}\text { NÚMERO DE EMPRESAS } \\
\text { QUE CONHECEM OS } \\
\text { MTF-Is }\end{array}$ & $\begin{array}{c}\text { NÚMERO DE EMPRESAS } \\
\text { QUE ADOTAM OS MTF-Is }\end{array}$ & $\begin{array}{c}\text { \% DE ADOÇÃO DAS } \\
\text { EMPRESAS QUE } \\
\text { CONHECEM OS MTF-Is }\end{array}$ \\
\hline Benchmarking & 7 & 7 & $100,00 \%$ \\
\hline Teste beta & 7 & 5 & $71,43 \%$ \\
\hline Análise conjunta & 0 & 0 & $0,00 \%$ \\
\hline Inquérito contextual & 4 & 2 & $50,00 \%$ \\
\hline DOE* & 7 & 5 & $71,43 \%$ \\
\hline DFSS** & 7 & 3 & $42,86 \%$ \\
\hline FMEA & 7 & 6 & $85,71 \%$ \\
\hline Grupo de foco & 5 & 2 & $40,00 \%$ \\
\hline QFD & 5 & 0 & $0,00 \%$ \\
\hline Parceria & 7 & 7 & $100,00 \%$ \\
\hline
\end{tabular}

* Projeto de Experiência (Design of Experiment - DOE).

** Projeto Seis Sigma (Design for Six Sigma - DFSS).

Fonte: Elaborado pelos autores com base em Thia et al. (2005).

Segundo as entrevistas realizadas por Thia et al. (2005) em seu estudo, ficou evidente que muitos fatores influenciam a adoção de MTF-I nas indústrias. Com base em estudos anteriores, assim como nas entrevistas, esses fatores podem ser classificados em duas categorias principais: internos e externos. Nesse sentido, os fatores internos identificados são: facilidade de uso, utilidade, tempo, custo monetário, flexibilidade e popularidade dos MTF-I. Já os fatores identificados como externos são: natureza do projeto, suporte da alta gestão, coesão da equipe, competência técnica, tamanho da empresa, natureza da indústria e diferenças culturais. Os autores desenvolveram uma proposição para ilustrar as relações de adoção dos MTF-I. Essa proposição é ilustrada na Figura 3. 


\section{FIGURA 3}

\section{RELAÇÕES DE ADOÇÃO DOS MTF-IS}

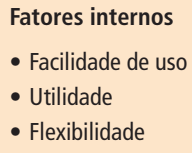

- Facilidade de uso

- Utilidade

- Flexibilidade

Fatores internos

- Tempo

- Custo monetário

- Popularidade

Fatores externos (projeto)

- Novidade do projeto

Fatores externos (organização)

- Suporte da alta gestão

- Coesão da equipe

- Competência técnica

- Tamanho da empresa

Fatores externos (setor)

- Natureza do setor

Fatores externos (cultura)

- Diferenças culturais

Fonte: Adaptada de Thia et al. (2005, p. 42I).

O trabalho de Igartua et al. (20I0) apresenta como os MTF-Is suportam uma estratégia de inovação aberta. Nesse sentido, os autores identificaram os grupos de MTF-Is utilizados em cada área da gestão da inovação, conforme apresenta o Quadro 9. 


\section{QUADRO 9}

GRUPOS DE MTF-IS UTILIZADOS NA GESTÃO DA INOVAÇÃO

\begin{tabular}{|c|c|c|c|c|c|c|c|c|c|c|c|}
\hline \multirow{2}{*}{ ÁREA DA GESTÃO DA INOVAÇÃO } & \multicolumn{11}{|c|}{ GRUPOS DE MTF-IS } \\
\hline & 1 & 2 & 3 & 4 & 5 & 6 & 7 & 8 & 9 & 10 & TOTAL \\
\hline Estratégia & & & $x$ & & & & & & & & 1 \\
\hline Gestão de portfólio & & & $x$ & & & $x$ & $x$ & & & & 3 \\
\hline Gestão de projeto & & & & & & $x$ & & & & & 1 \\
\hline Liderança e cultura & & & & $x$ & & & & & & & 1 \\
\hline Recursos humanos & $x$ & & & $x$ & & & & & & & 2 \\
\hline Relações externas & & & & & & & & $x$ & & & 1 \\
\hline Design organizacional & & & & $x$ & & & & & $x$ & & 2 \\
\hline Processos & $x$ & & & & & & $x$ & & & & 2 \\
\hline Indicadores & & & & & & & & & & & 0 \\
\hline Mercado & & & & & $x$ & & & & & & 1 \\
\hline Recursos & & & & & & & $x$ & & & & 1 \\
\hline Proteção & & $x$ & & & & & & & & & 1 \\
\hline Conhecimento & & & & & & & & & & $x$ & 1 \\
\hline Tecnologia & & $x$ & $x$ & & & & & & & & 2 \\
\hline Total & 2 & 2 & 3 & 3 & 1 & 2 & 3 & 1 & 1 & 1 & \\
\hline
\end{tabular}

Nota: Os grupos de MTF-Is são: I. desenvolvimento de criatividade, 2. gestão de tecnologias, 3. gestão estratégica, 4. gestão de recursos humanos, 5. business intelligence, 6. gestão de projetos inovadores, 7. desenvolvimento de novos produtos e processos, 8. cooperação e relacionamentos, 9. gestão de design e Io. gestão do conhecimento.

Fonte: Elaborado pelos autores com base em Igartua et al. (2010, p. 45).

Dessa forma, Igartua et al. (2010) identificaram os MTF-Is utilizados na Orona, empresa onde foi realizado o estudo de caso, bem como os impactos nos objetivos dela. O Quadro Io apresenta esse resultado. 
QUADRO IO

MTF-IS E IMPACTOS NOS OBJETIVOS DA ORONA

\begin{tabular}{|c|c|c|c|c|c|}
\hline \multirow{2}{*}{$\begin{array}{l}\text { GRUPOS } \\
\text { DE MTF-I }\end{array}$} & \multirow{2}{*}{ MTF-IS IDENTIFICADOS } & \multicolumn{4}{|c|}{ IMPACTO NOS OBJETIVOS DA ORONA } \\
\hline & & RELACIONAMENTO & ALINHAMENTO & FINANÇAS & QUALIDADE \\
\hline \multirow{4}{*}{1} & Formação de equipes & Alto & Alto & Baixo & Médio \\
\hline & Networking & Alto & Alto & Baixo & Médio \\
\hline & Projetos colaborativos & Alto & Alto & Alto & Alto \\
\hline & Terceirização & Baixo & Baixo & Alto & Médio \\
\hline 2 & $\begin{array}{l}\text { Pesquisa de desenvolvimento } \\
\text { (P\&D) em marketing }\end{array}$ & Baixo & Alto & Baixo & Alto \\
\hline \multirow{3}{*}{3} & Brainstorming & Baixo & Alto & Baixo & Alto \\
\hline & Workshops de criatividade & Médio & Alto & Baixo & Alto \\
\hline & Painéis de experts & Baixo & Baixo & Baixo & Médio \\
\hline 4 & $\begin{array}{l}\text { Gestão de direitos de } \\
\text { propriedade intelectual }\end{array}$ & Baixo & Baixo & Alto & Médio \\
\hline 5 & Gestão de portfólio & Médio & Alto & Alto & Alto \\
\hline \multirow{3}{*}{6} & Vigilânica tecnológica & Médio & Alto & Baixo & Médio \\
\hline & Roadmapping & Alto & Alto & Baixo & Alto \\
\hline & Técnica de cenário & Alto & Alto & Baixo & Alto \\
\hline \multirow{2}{*}{7} & Análise de patentes & Baixo & Baixo & Baixo & Médio \\
\hline & Business intelligence & Baixo & Baixo & Baixo & Médio \\
\hline \multirow{2}{*}{8} & Análise de investimentos & Baixo & Baixo & Alto & Baixo \\
\hline & $P \& D$ em finanças & Baixo & Médio & Alto & Baixo \\
\hline 9 & Empresa virtual & Alto & Médio & Baixo & Baixo \\
\hline
\end{tabular}

Nota: Os grupos de MTF-Is são: I. cooperação e relacionamentos, 2. abordagem de gestão de interfaces, 3. desenvolvimento de criatividade, 4 . gestão de direitos de propriedade intelectual, 5 . gestão de pesquisa e desenvolvimento, 6 . gestão de tecnologias, 7. inteligência de mercado, 8. inovação em finanças, 9. organizacionais.

Fonte: Elaborado pelos autores com base em Igartua et al. (2010).

O resultado do estudo de caso realizado por Igartua et al. (20I0) demonstrou que os MTF-Is podem ajudar as empresas a gerir a inovação, adaptar-se às circuns- 
tâncias mutáveis e conhecer os desafios do mercado de uma forma sistemática. $\mathrm{Na}$ Orona, empresa pesquisada, os MTF-Is desempenham um papel importante no sentido de facilitar uma estratégia de inovação aberta, especialmente na construção e melhoria da rede. A adoção dos MTF-Is resultou no alinhando dos membros da empresa aos objetivos comuns, na melhora da qualidade dos projetos definidos e no estabelecimento de sistemas de financiamento mais eficientes.

O foco do trabalho de Olsen e Welo (20II) foi maximizar a inovação de produtos por meio da aplicação adaptativa de MTF-Is centrados no usuário, para definir o valor do cliente e atender aos requisitos dele. Para isso, os autores utilizaram levantamentos baseados na web, em entrevistas, observações e oficinas. Além disso, utilizaram outro MTF-I, chamado paleta de design, para avaliar esses MTF-Is. A paleta de design é um MTF-I cujo objetivo é organizar, visualizar e comunicar os resultados da investigação em um contexto de negócios. Com essa avaliação, foi possível classificar os MTF-Is de acordo com a eficiência e o tipo de informação gerada. Nesse sentido, o estudo mostrou que o levantamento baseado na web possui alta eficiência, porém produz informações superficiais. Já as oficinas têm baixa eficiência, mas geram informações com alto nível de profundidade, conforme mostra a Figura 4.

\section{FIGURA 4}

\section{MTF-IS CLASSIFICADOS DE ACORDO COM A EFICIENCIA} E O TIPO DE INFORMAÇÃO PRODUZIDA

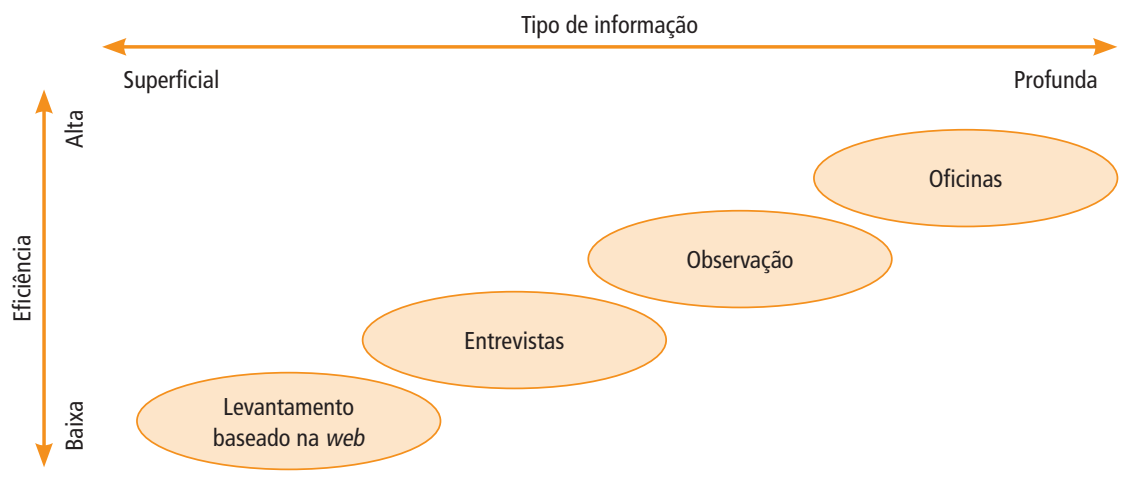

Fonte: Olsen e Welo (20II, p. I87).

Os resultados indicam que os MTF-Is que revelam informações emocionalmente relacionadas com o cliente (oficinas e observação) são intensivos em recursos e fornecem menor quantidade de informações diretamente aplicadas à equipe de desenvolvimento do produto. O oposto é o caso de MTF-Is que fornecem informações mais funcionalmente relacionadas (pesquisa baseada na web 
e entrevista). A conclusão geral é que os últimos MTF-Is são mais adequados para a melhoria do produto, enquanto os primeiros podem fornecer informações valiosas para a criação de produtos mais diferenciados.

Em relação ao trabalho de Keltsch et al. (2OII), foi analisado somente o resumo, pois o artigo completo não estava disponível. Nesse sentido, o trabalho trata do processo pelo qual os gerentes implementam os MTF-Is de gestão tecnológica dentro das organizações. Os resultados desse estudo demonstraram que a aplicação de MTF-I é desencadeada por diversos eventos e quatro fases do processo estudado, e pela configuração, execução e reconfiguração dos MTF-Is.

O último trabalho analisado foi de Laurenti et al. (20I2) que trata da avaliação da aplicação dos MTF-Is FMEA e DRBFM no processo de desenvolvimento de produtos em uma empresa brasileira de autopeças. Nesse sentido, os autores identificaram alguns MTF-Is que auxiliam a utilização daqueles em estudo, bem como a frequência de utilização, conforme apresenta o Quadro II.

\section{QUADRO II}

\section{FERRAMENTAS UTILIZADAS PARA AUXILIAR O PROCESSO DE DESENVOLVIMENTO DE PRODUTOS}

MTF-I

FREQUÊNCIA DE UTILIZAÇÃO

NUNCA ÀS VEZES QUASE SEMPRE SEMPRE

\begin{tabular}{llc}
\hline Análise da árvore de falhas & $X$ \\
\hline Software específico & $X$ & $X$ \\
\hline Diagrama de Ishikawa (espinha de peixe) & $X$ \\
\hline Diagrama de blocos (DFMEA*/DRBFM**) & $X$ \\
\hline Diagrama de fluxo do processo (PFMEA) & $X$ \\
\hline Gráfico de Pareto & $X$ \\
\hline Checklist de possíveis falhas & X \\
\hline \begin{tabular}{l} 
8D (oito disciplinas) \\
\hline DFMA (projeto para manufatura e montagem)
\end{tabular} \\
\hline $\begin{array}{l}\text { * Para cada um dos dois tipos de falhas, é utilizado um tipo de FMEA: o FMEA de produto (Design } \\
\text { FMEA - DFMEA) ou o FMEA de processo (Process FMEA - PFMEA). Um DFMEA enfoca modos } \\
\text { de falha causados pelas deficiências de projeto do produto (design), e um PFMEA enfoca modos de } \\
\text { falha causados por deficiências de processo de fabricação ou montagem. }\end{array}$
\end{tabular}

** Revisão de Projeto Baseada nos Modos de Falha (Design Review Based on Failure Mode - DRBFM).

Fonte: Adaptado de Laurenti et al. (2012, p. 849). 
Os resultados da avaliação de Laurenti et al. (20I2) sugerem que o sucesso da aplicação dos MTF-Is FMEA e DRBFM, na unidade de negócio, é devido à combinação de provisão de recursos, trabalho em equipe multidisciplinar, formação de competências (treinamento), definição de procedimentos, aplicação integrada com outros MTF-Is no processo de desenvolvimento de produtos e, sobretudo, não consideração da aplicação deles como uma atividade pró-forma.

Com base na análise dos artigos levantados, verifica-se que não existe uma convergência de resultados. Entretanto, percebe-se que a utilização de MTF-Is é importante para a competitividade das empresas, pois ajudam a gerenciar a inovação e solucionar problemas.

A próxima seção apresenta as considerações finais deste trabalho e as indicações para estudos futuros, como foi apontado por alguns dos autores analisados.

\section{CONSIDERAÇÕES FINAIS}

O presente trabalho analisou as pesquisas qualitativas empíricas referentes aos MTF-Is. Os artigos analisados foram obtidos a partir de um levantamento sistemático em duas bases de dados: Scopus e Web of Science. Após a eliminação dos artigos repetidos e não relevantes para o estudo, chegou-se a um total de dez artigos, dos quais um não estava disponível na íntegra. Em seguida, analisou-se as referências desses nove artigos em busca de outros trabalhos que não estivessem cadastrados nas bases de dados utilizadas. Nesse sentido, não foram encontrados outros artigos importantes, além dos já levantados.

Considera-se que este estudo contribui para: I. evidenciar a importância dada pelas pesquisas para os MTF-Is; 2. auxiliar no avanço científico do tema a partir da construção de um mapa atual das pesquisas; 3 . fornecer um panorama geral acerca do tema, especialmente dos trabalhos com abordagem qualitativa empírica. Nesse sentido, espera-se que o trabalho possa ser útil para aqueles que desejam ter uma visão ampla em relação ao tema, bem como favorecer e apontar novos caminhos de investigação em novas pesquisas.

Os trabalhos aqui analisados sugerem que o uso de MTF-I pode impactar positivamente o desempenho dos projetos de inovação, apesar de poucos apresentarem uma relação mais clara de causa e efeito dessa utilização (por exemplo, Igartua et al., 20ıо). A maioria dos estudos apenas descreve a utilização de MTF-I (por exemplo, Lemos \& Porto, I998; Libutti, 2000; Ghaemmaghami \& Bucciarelli, 2003; Olsen \& Welo, 20II; Laurenti et al., 20I2). Outros classificam os MTF-Is em grupos de acordo com os objetivos de cada um (por exemplo, Lemos \& Porto, I998; Lichtenthaler, 2005; Scozzi et al., 2005; Igartua et al., 
20Io). Outra observação importante diz respeito à não mensuração da intensidade com que cada MTF-I foi utilizado nos trabalhos. Além disso, cada estudo tem resultados individuais, o que dificulta o agrupamento dos dados e a comparação entre eles.

Muitas lacunas de pesquisa ainda parecem existir, uma vez que se identificou que os trabalhos analisados apresentam poucos pontos de convergência. Desses poucos, a maior parte indica que a utilização de MTF-I pelas empresas é para solução de problemas (por exemplo, Scozzi et al., 2005). Assim, são escassos os estudos que investiguem a relação de causa e efeito do uso de MTF-I. Essa dificuldade pode se tornar ainda maior quando se trata de estudos qualitativos, os quais apresentam resultados mais difíceis de validação interna e externa. Nesse sentido, Laurenti et al. (20I2) destacam a necessidade da realização da mesma avaliação em outras empresas, de tamanhos e setores diferentes e iguais, por meio de estudo de múltiplos casos. Assim, além de análise intracaso, poderia ser realizada uma análise intercaso, para verificação de relações causais e generalização de conclusões.

Futuras pesquisas poderão abordar de forma mais profunda tanto a utilização de MTF-I quanto o impacto causado no desempenho em inovação nas empresas. Pode-se ainda afirmar que existem várias possibilidades de entrar em mais detalhes sobre a escolha dos MTF-Is, ou seja, poderiam ser criados conjuntos de métodos para indústrias específicas ou para pequenas e médias empresas. Além disso, a implementação de MTF-Is específicos também carece de mais pesquisas (Lichtenthaler, 2005). Lichtenthaler (2005) ressalta que são necessários estudos para identificar um meio sistemático para definir qual o melhor MTF-I para um determinado contexto organizacional. Segundo Thia et al. (2005), uma seleção mais ampla de empresas que vão desde pequenas empresas privadas até empresas multinacionais pode ser considerada. Outro ponto importante diz respeito aos fatores externos que não são profundamente examinados no estudo. No entanto, muitas análises preliminares desses fatores foram realizadas por muitos outros pesquisadores. A adoção de uma abordagem quantitativa em uma amostra maior para testar todas as proposições pode ser uma abordagem mais econômica de tempo. A pesquisa pode também se estender até regiões além do contexto local. Dessa forma, ele também pode ajudar a identificar e comparar a adoção de MTF-I em uma base regional. Com esses resultados, podem ser desenvolvidas melhores diretrizes e metodologias para facilitar um sistema mais eficaz de implementação de MTF-I. Por fim, sugere-se que estudos futuros sejam feitos a partir de uma abordagem mais sistemática para realizar a coleta de dados, dando maior credibilidade e rigor científico ao estudo. 


\section{ANALYSIS OF EMPIRICAL QUALITATIVE ARTICLES ON METHODS, TECHNIQUES, AND TOOLS FOR INNOVATION}

\section{ABSTRACT}

Innovation has been regarded as a crucial element to create long-term competitive advantage in companies. However, encouraging and supporting the innovation process still remains a challenge. In turn, the effective use of methods, techniques, and tools for innovation may be a significant factor to support the innovation management process, increasing its chances of success. Thus, this study consisted in a qualitative analysis of empirical articles related to diffusion and adoption of innovation methods, tools, and techniques. The articles analyzed herein were obtained through a systematic search conducted through 2 databases: Scopus and Web of Science. This systematic search consisted of 7 steps: identification of keywords; search in the databases; filtering of publications; standardization; classification of articles; inclusion of relevant publications; analysis of articles. As a result, we obtained a corpus with ten publications (nine fully available and I only as an abstract), where, initially, the main articles, authors, countries, and journals that have published more and the more frequently used keywords were identified. Later, through the analysis, an overview of the empirical qualitative investigations related to the theme was obtained, given the importance of MTF-I for the innovation process of companies. It is noteworthy that there are still few papers addressing this theme with the approach chosen in this research, and the results are not converging. There was a great diversity of methods, techniques, and tools for innovation used by the companies studied. Yet, we found that the use of MTF-I is significant to increase competitiveness and they are mainly used to solve problems in organizations. The need for further studies aimed to systematize decision making about the choice of an MTF-I for a particular organizational context was noticed. Thus, it is expected that this paper can serve as a basis for future research on the theme.

\section{KEYWORDS}

Innovation. Methods, techniques, and tools for innovation. MTF-I. Systematic survey. Qualitative methods. 


\section{ANÁLISIS DE LOS ARTIÍCULOS CUALITATIVOS EMPÍRICOS SOBRE MÉTODOS, TÉCNICAS Y HERRAMIENTAS PARA INNOVACIÓN}

\section{RESUMEN}

La innovación ha sido considerada como esencial para crear una ventaja competitiva a largo plazo en el elemento empresarial. Sin embargo, para fomentar y apoyar el proceso de innovación sigue siendo un reto. A su vez, el uso efectivo de los métodos, técnicas y herramientas para la innovación puede ser un apoyo importante para el proceso de gestión de la innovación, aumentando sus posibilidades de factor de éxito. En este sentido, este estudio consiste en un análisis cualitativo de los artículos empíricos relacionados con la difusión y la adopción de métodos, herramientas y técnicas para la innovación. Los artículos analizados se obtuvieron a través de un estudio sistemático realizado a partir de las bases de datos: Scopus y Web of Science. Esta encuesta sistemática consistió en siete pasos: identificación de palabras clave; búsquedas en bases de datos; filtrado de las publicaciones; normalización; clasificación de los artículos; inclusión de las publicaciones pertinentes; análisis de los artículos. Como resultado se obtuvo un corpus de diez artículos (nueve disponible y listado completo y un resumen), en el que se identificaron inicialmente los principales artículos, autores, países y revistas que han publicado más y más palabras clave utilizadas. Más tarde, a partir del análisis se permitió una visión general de la investigación cualitativa empírica relacionada con el tema, dada la importancia de MTF-I para el proceso de innovación de las empresas. Es de destacar que todavía hay pocos trabajos sobre este tema con el enfoque utilizado en este estudio, más allá de los resultados no están convergiendo. Había una gran diversidad de métodos, técnicas y herramientas para la innovación utilizado por las empresas estudiadas. Por otra parte, se encontró que el uso de MTF-I es importante para aumentar la competitividad y se utilizan principalmente para resolver problemas en las organizaciones. Se observó la necesidad de más estudios con el objetivo de sistematizar la toma de decisiones sobre la elección de un MTF-I para un contexto organizativo concreto. Por lo tanto, se espera que este trabajo pueda servir de base para futuras investigaciones sobre el tema.

\section{palabras clave}

Innovación. Métodos, técnicas y herramientas para innovación. MTF-I. Levantamiento sistemático. Métodos cualitativos. 


\section{REFERÊNCIAS}

Baregheh, A., Rowley, J., \& Sambrook, S. (2009). Towards a multidisciplinary definition of innovation. Management Decision, 47(8), I323-1339.

Blocker, C. P., Flint, D. J., Myers, M. B., \& Slater, S. F. (2OII). Proactive customer orientation and its role for creating customer value in global markets. Journal of the Academy of Marketing Science, 39(2), 216-233.

Brady, T., Rush, H., Hobday, M., Davies, A., Probert, D., \& Banerjee, S. (I997). Tools for technology management: an academic perspective. Technovation, 17(8), 4I7-426.

Chai, K. H., \& Xin, Y. (2006). The application of new product development tools in industry: the case of Singapore. IEEE Transactions on Engineering Management, 53(4), 543-554.

Chiesa, V., \& Masella, C. (I996). Searching for an effective measure of R\&D performance. Management Decision, 34(7), 49-57.

Cooper, R. G., \& Kleinschmidt, E. J. (1986). An investigation into the new product process: steps, deficiencies, and impact. Journal of Product Innovation Management, 3(2), 7I-85.

Coulon, M., Ernst, H., Lichtenthaler, U., \& Vollmoeller, J. (2009). An overview of tools for managing the corporate innovation portfolio. International Journal of Technology Intelligence and Planning, $5(2), 22 \mathrm{I}-239$.

D'Alvano, L., \& Hidalgo, A. (20I2). Innovation management techniques and development degree of innovation process in service organizations. $R$ and $D$ Management, 42(I), 60-70.

Fleisher, C. S. (2006). Assessing the tools and techniques enterprises use for analysing Innovation, Science and Technology (IS\&T) factors: are they up to the task? International Journal of Technology Intelligence and Planning, 2(4), 380-403.

Flynn, M., Dooley, L., O’Sullivan, D., \& Cormican, K. (2003). Idea management for organisational innovation. International Journal of Innovation Management, 7(4), 4I7-442.

Ghaemmaghami, S., \& Bucciarelli, L. (2003). Structured methods in product development. International Journal of Engineering Education, 19(I), I32-I4I.

Graner, M., \& Mißler-Behr, M. (20I2). The use os methods in new product development - a review of empirical literature. International Journal of Product Development, 16(2), I58-184.

Hidalgo, A., \& Albors, J. (2008). Innovation management techniques and tools: a review from theory and practice. $R$ and $D$ Management, 38(2), II3-I27.

Igartua, J. I., Garrigós, J. A., \& Hervas-Oliver, J. L. (20I0). How innovation management techniques support an open innovation strategy. Research Technology Management, 53(3), 4I-52.

Keltsch, J. N., Probert, D., \& Phaal, R. (20II). A process for configuring technology management tools. International Journal of Technology Intelligence and Planning, 7(3), I8I-200.

Laurenti, R., Rozenfeld, H., \& Franieck, E. K. (20I2). Avaliação da Aplicação dos Métodos FMEA e DRBFM no Processo de Desenvolvimento de Produtos em uma Empresa de Autopeças. Gestão e Produção, 19(4), 84I-855.

Lee, S., Mortara, L., Kerr, C., Phaal, R., \& Probert, D. (20I2). Analysis of document-mining techniques and tools for technology intelligence: discovering knowledge from technical documents. International Journal of Technology Management, 6o(I-2), I30-156. 
Lemos, A. D., \& Porto, A. C. (1998). Technological forecasting techniques and competitive intelligence: tools for improving the innovation process. Industrial Management and Data Systems, 98(7), 330-337.

Libutti, L. (2000). Building competitive skills in small and medium-sized enterprises through innovation management techniques: overview of an Italian experience. Journal of Information Science, 26(6), 413-4I9.

Lichtenthaler, E. (2005). The choice of technology intelligence methods in multinationals: towards a contingency approach. International Journal of Technology Management, 32(3-4), 388-407.

Mahajan, V., \& Wind, J. (I992). New product models: Practice, shortcomings and desired improvements. The Journal of Product Innovation Management, 9(2), I28-I39.

Mattar, F. N., Oliveira, B. A. C. de, Queiroz, M. J. de, \& Motta, S. L. S. (2009). Gestão de produtos, serviços, marcas e mercados: estratégias e ações para alcançar e manter-se Top of Market. São Paulo: Atlas. Nijssen, E. J., \& Frambach, R. T. (2000). Determinants of the adoption of new product development tools by industrial firms. Industrial Marketing Management, 29(2), I2I-I3I.

Nijssen, E. J., \& Lieshout, K. F. M. (I995). Awareness, use and effectiveness of models and methods for new product development. European Journal of Marketing, 29(10), 27-44.

Olsen, T. O., \& Welo, T. (2OII). Maximizing product innovation through adaptive application of user-centered methods for defining customer value. Journal of Technology Management and Innovation, 6(4), I72-I9I.

Phaal, R., Farrukh, C. J. P., \& Probert, D. R. (2006). Technology management tools: concept, development and application. Technovation, 26(3), 336-344.

Phaal, R., Kerr, C., Oughton, D., \& Probert, D. (20I2). Towards a modular toolkit for strategic technology management. International Journal of Technology Intelligence and Planning, 8(2), I6I-I8I. Scozzi, B., Garavelli, C., \& Crowston, K. (2005). Methods for modeling and supporting innovation processes in SMEs. European Journal of Innovation Management, 8(I), I2O-I37.

Shehabuddeen, N., Probert, D., Phaal, R., \& Platts, K. (I999). Representing and approaching complex management issues: part I - role and definition [Working Paper $\mathrm{N}^{\circ} \mathrm{CTM} 2000 / 03$ ]. Centre for Technology Management Working Paper Series. Cambridge, I-2O.

Thia, C. W., Chai, K. H., Bauly, J., \& Xin, Y. (2005). An exploratory study of the use of quality tools and techniques in product development. TQM Magazine, 17(5), 406-424.

Tian, X., \& Wang, T. Y. (2OI4). Tolerance for failure and corporate innovation. The Review of Financial Studies, 27(I), 2II-255.

Walker, R. (2006). Innovation type and diffusion: an empirical analysis of local government. Public Administration, 84(2), 3II-335.

Yeh, T. M., Pai, F. Y., \& Yang, C. C. (2010). Performance improvement in new product development with effective tools and techniques adoption for high-tech industries. Quality and Quantity, 44(I), I3I-I52. 